\title{
RELIABILITY OF THE
}

\section{MANITOBA MENTAL HEALTH MANAGEMENT INFORMATION SYSTEM}

FOR RESEARCH

BY

\section{J. RENEE ROBINSON}

\author{
A thesis \\ Submitted to the Faculty of Graduate Studies \\ in Partial Fulfilment of the Requirements \\ for the Degree of
}

MASTER OF SCIENCE

\author{
Department of Community Health Sciences \\ University of Manitoba \\ Winnipeg, Manitoba
}

(c) August, 1996 
The author has granted an irrevocable non-exclusive licence allowing the National Library of Canada to reproduce, loan, distribute or sell copies of his/her thesis by any means and in any form or format, making this thesis available to interested persons.
The author retains ownership of the copyright in his/her thesis. Neither the thesis nor substantial extracts from it may be printed or otherwise reproduced without his/her permission.
L'auteur a accordé une licence irrévocable et non exclusive permettant à la Bibliothèque nationale du Canada de reproduire, prêter, distribuer ou vendre des copies de sa thèse de quelque manière et sous quelque forme que ce soit pour mettre des exemplaires de cette thèse à la disposition des personnes intéressées.

L'auteur conserve la propriété du droit d'auteur qui protège sa thèse. Ni la thèse ni des extraits substantiels de celle-ci ne doivent être imprimés ou autrement reproduits sans son autorisation. 
ime

isertation Abstracts International and Masters Abstracts International are arranged by broad, general subject categories. ase select the one subject which most nearly describes the content of your dissertation or thesis. Enter the corresponding ir-digit $\alpha d z$ in the spaces provided.

Heath Sciences Mental Heaith SUBJECT TERM

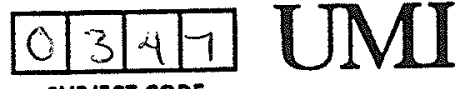

SUBJECT CODE

\section{bject Categories}

\section{IE HUMANITIES AND SOCLAL SCIENCES}

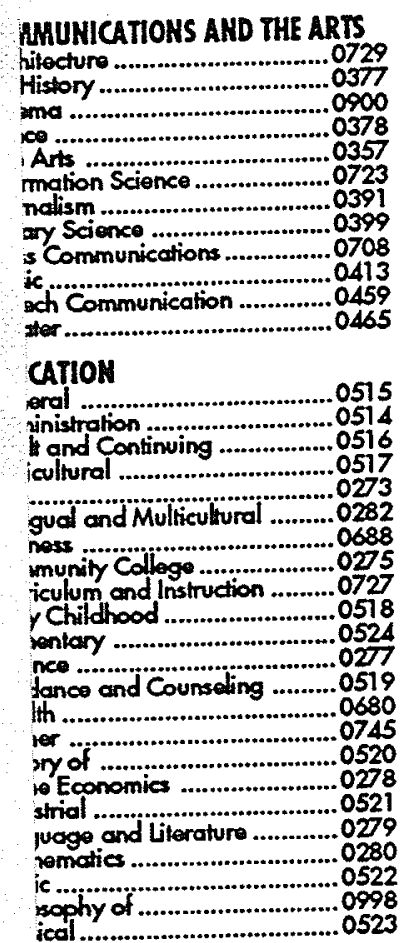

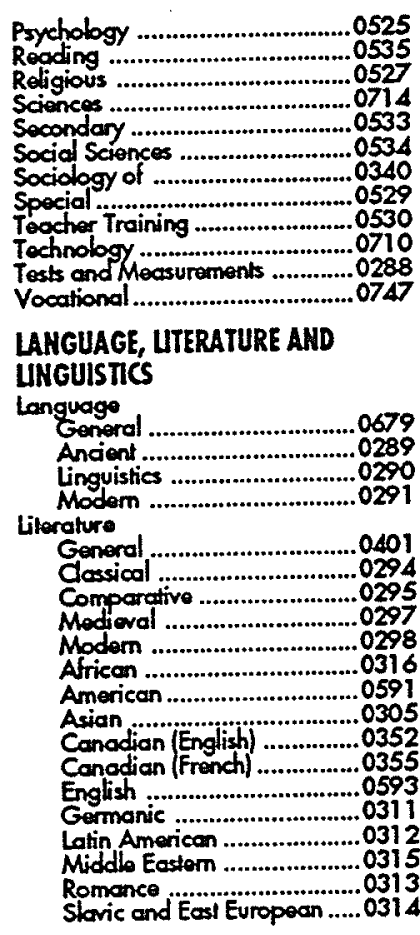

\section{ENGINEERING}

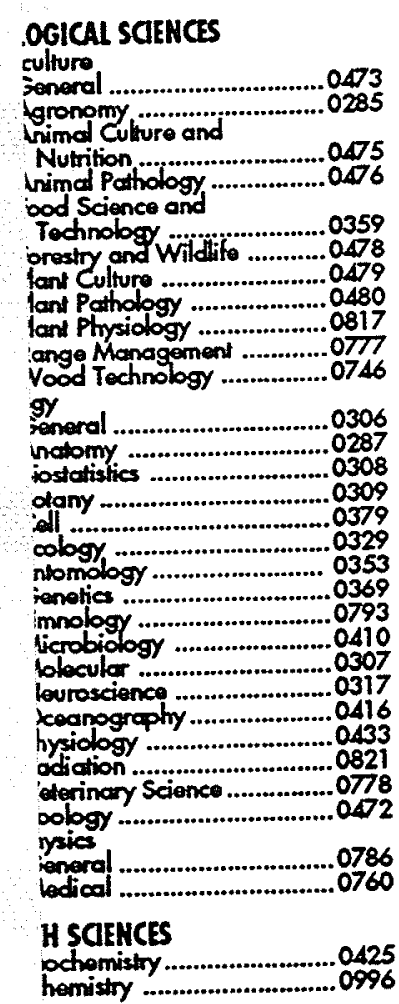

PHILOSOPHY, RELIGION AHD

THEOLOGY

Philosophy ................................0422

Religion

0318

Genaral .............................0321

Biblicd Studies ......................03219

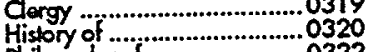

Philosophy of .......................0322

Thodogy .....................................0469

SOCIAL SCIEHCES

American Studies ........................0323

Anthropology

Archooology ……….............0324

Cultural ................................0326

Business Administration

General ..............................0310

Accounting ...........................0272

Banking ............................077

Maragement ...........................0453

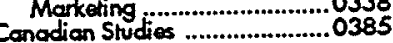

Conadian Stud

General ................................0501

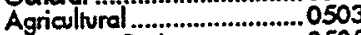

Commerce-Business ..............0505

Finance ………...................0508

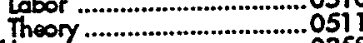

Follore

Goography.................................036

History

.0578
History ……...............................0510

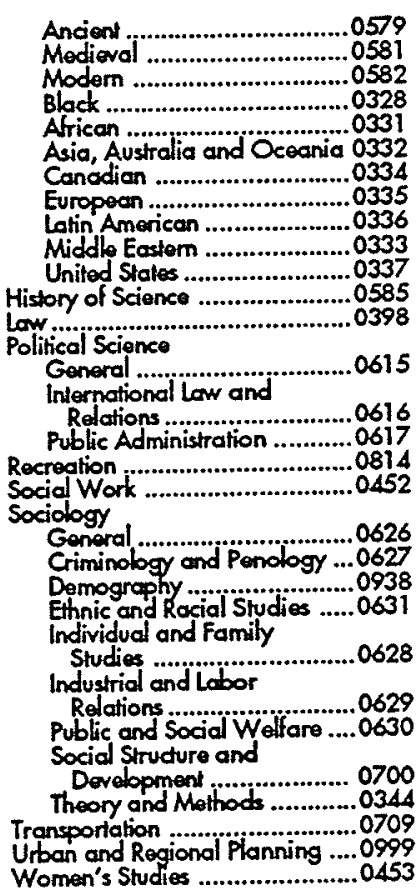

Speoch Pathology ................0460 Toxicology ...............................0383 Home Economics ........................0386 PHYSICAI SOENCES

\section{Pure Sciences}

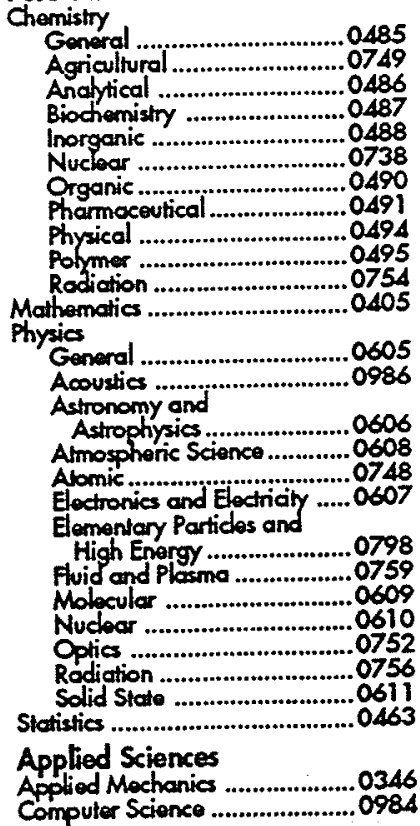

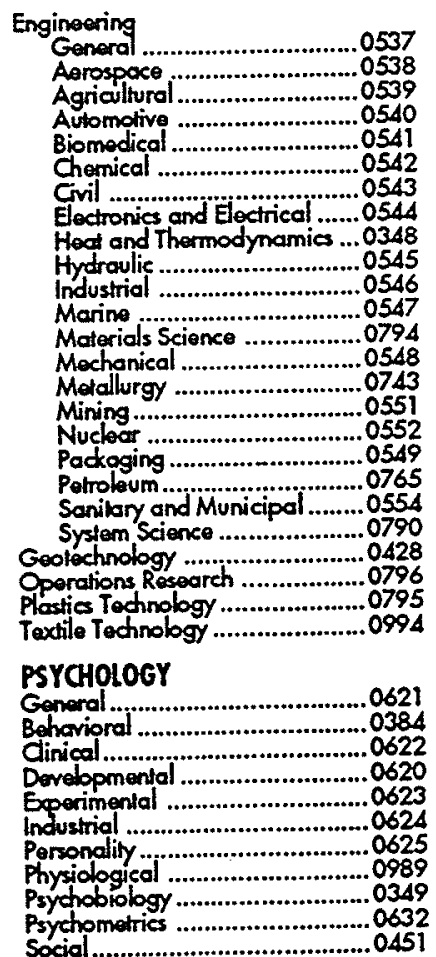

Engineering

Aorospoce .......

Agriallural .........................054

Chenical ..............................0542

Givi …….......054

Hod and Thermodynamics ... 0348

industrial .....................................0546

...........059

Mechanical .........................0548

Metalurgy ..........................0743

Mining ..................................055

Padogging ............................0549

Sanilary and Municipol .........055

System Science

Geotechnology ..........................0428

Plastios Todinology .......................0795

Textile Technology .........................0994

PSYCHOLOGY

Modicine and Surgary ..........0564

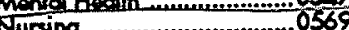

Nutrition .........................0570

Obstetria and Gypocology ..0380

Oceupationd Hoalth and

Therapy aphi.........................035

Pothology .............................0571

Prarmoodogy ........................0419

Prarmacy ..........................0572

Physical horopy ..................038

Radidogy ...............................057 


\section{THE UNIVERSITY OF MLANTTOBA}

\section{FACULTY OF GRADUATE STUDIES}

\section{COPYRIGHT PERUISSION}

RELIABILITY OF THE MANITOBA MENTAL HEALTH

MANAGEMENT INFORMATION SYSTEM FOR RESEARCH

BY

J. RENEE ROBINSON A Thesis/Practicum submitted to the Faculty of Graduate Studies of the University of Manitoba in partial
fulfillment of the requirements for the degree of

MASTER OF SCIENCE

J. Renee Robinson 01996

Permission has been granted to the LIBRARY OF THE UNTVERSITY OF MANITOBA to lend or sell copies of this thesis/practicum, to the NATIONAL LIBRARY OF CAN.ADA to microfilm this thesis'practicum and to lend or sell copies of the film, and to UNTVERSITY MIICROFILMS INC. to publish an abstract of this thesis/practicum..

This reproduction or copy of this thesis has been made available by authority of the copyright owner solely for the purpose of private study and research, and may only be reproduced and copied as permitred by copyright laws or with express written authorization from the copyright owner. 


\section{TABLE OF CONTENTS}

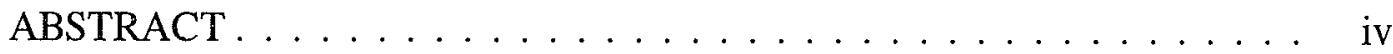

ACKNOWLEDGEMENTS . . . . . . . . . . . . . . vi

LIST OF TABLES $\ldots \ldots \ldots \ldots \ldots \ldots \ldots \ldots \ldots$ vii

LIST OF ABBREVIATIONS $\ldots \ldots \ldots \ldots \ldots \ldots \ldots$ ix

INTRODUCTION $\ldots \ldots \ldots \ldots \ldots \ldots \ldots \ldots \ldots \ldots$

REVIEW OF THE LITERATURE . . . . . . . . . . . . . . . 3

Applications of Mental Health Information Systems . . . . . . . 3

Mental Health Information Systems and Case Registers in

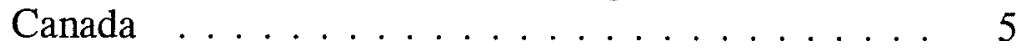

Strengths and Limitations of Administrative Data . . . . . . . . 8

Reliability Studies . . . . . . . . . . . . . . . . . . . 9

Factors Influencing Data Quality . . . . . . . . . . . . 11

Confidentiality . . . . . . . . . . . . . . . . 14

BACKGROUND ....................... 15

Manitoba Health Research Data Base . . . . . . . . . . . . 15

Provincial Mental Health Services . . . . . . . . . . . . . . 17

MHMIS Data . . . . . . . . . . . . . . . . . . . 18

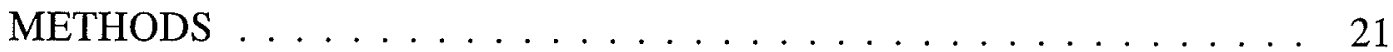

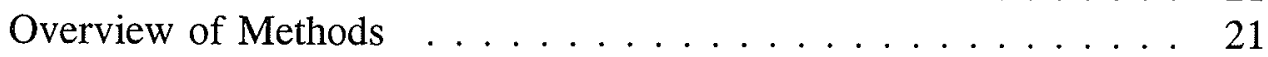

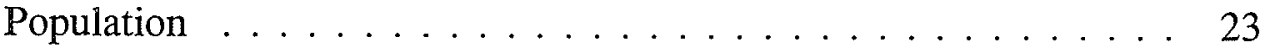

Chart Reviews .................. . . 23

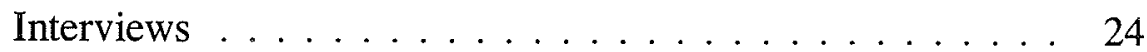

Sample . . . . . . . . . . . . . . . . . 25

Chart Reviews . . . . . . . . . . . . . 25

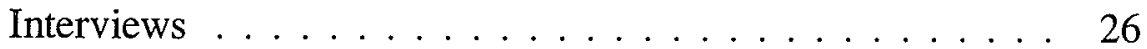

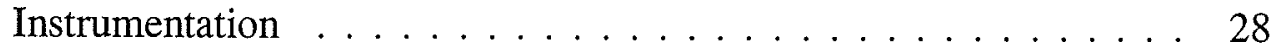

Chart Reviews . . . . . . . . . . . . . . 28

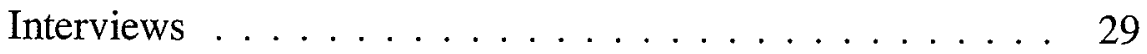

Implementation $\ldots \ldots \ldots \ldots \ldots \ldots$

Analysis . . . . . . . . . . . . . . . . . . 32

Chart Reviews . . . . . . . . . . . . . 32

Interviews $\ldots \ldots \ldots \ldots \ldots \ldots \ldots \ldots \ldots \ldots$

Ethics . . . . . . . . . . . . . . . . . . . 34

Generalizability Analysis $\ldots \ldots \ldots \ldots \ldots \ldots \ldots \ldots$

Detailed Methods . . . . . . . . . . . . . . . . . . 38 
Results .......................... 39

DETAILED METHODS AND RESULTS . . . . . . . . . . . . . . . . . . . 40

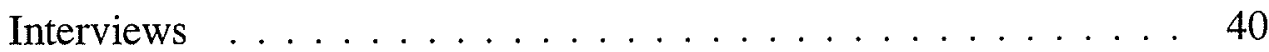

Flow of Information . . . . . . . . . . . . 41

Inpatient Services . . . . . . . . . . . . . . . 41

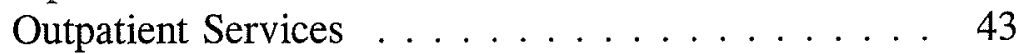

Regional Services . . . . . . . . . . . . . . . . . . 44

Accuracy of MHMIS Information . . . . . . . . . . . . . . 44

Demographic Information . . . . . . . . . . . . . . 44

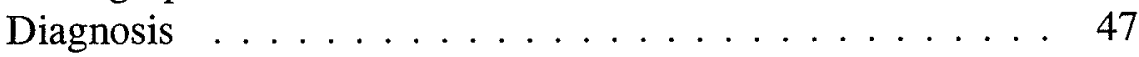

Contact Information . . . . . . . . . . . . . . . 54

Therapy Information . . . . . . . . . . . . . . 61

Open Date and Close Date . . . . . . . . . . . . . 62

Change of Status Information $\ldots \ldots \ldots . \ldots 67$

Uses of MHMIS Information . . . . . . . . . . . . . 69

Other Issues . . . . . . . . . . . . . . . . . . . . 74

Chart Reviews . . . . . . . . . . . . . . . . . . . 77

Demographic Data . . . . . . . . . . . . . . 78

Detailed Methods for Demographic Information . . . 79

Results for Demographic Information . . . . . . . . 82

Required information . . . . . . . . 82

Optional information ............ 85

Issues Regarding Demographic Information . . . . 85

Diagnosis ......................... 88

Detailed Methods for Diagnosis . . . . . . . 90

Results for Diagnosis . . . . . . . . . . . . . . . . 94

Primary diagnosis . . . . . . . . . . . . . 94

Other diagnoses . . . . . . . . . . . . . . . . . . . 98

Issues Regarding Diagnosis . . . . . . . . . . . . . 99

Contact Information . . . . . . . . . . . . . . . . . 101

Detailed Methods for Contact Information . . . . . 102

Results for Contact Information . . . . . . . . . . . . . 104

Issues Regarding Contact Information . . . . . . . . 107

Open and Close Dates . . . . . . . . . . . . . . . . 110

Detailed Methods for Open and Close Dates . . . . . 110

Results for Open and Close Dates . . . . . . . . . . 112

Issues Regarding Open and Close Dates . . . . . . . . 115

Overall Reliability of MHMIS and Chart Data . . . . . . 118 
Detailed Methods for Overall Reliability . . . . . 118

Results for Overall Reliability . . . . . . . . . . . 119

Issues Regarding Overall Reliability . . . . . . . . 119

ISSUES AFFECTING THE INFORMATION AS A WHOLE $\ldots \ldots \ldots 122$

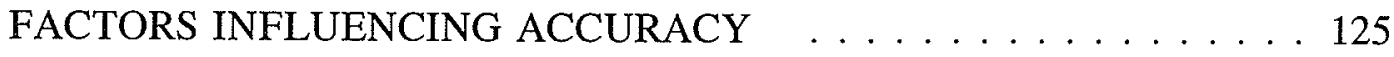

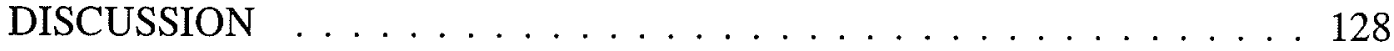

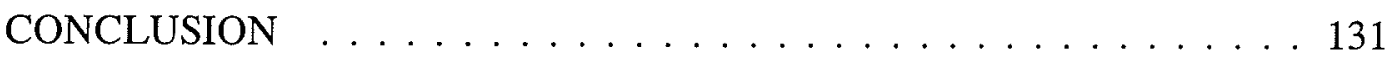

REFERENCES. . . . . . . . . . . . . . . . . . . . . 132

GLOSSARY . . . . . . . . . . . . . . . . . . . . . . 141

Appendix A: MHMIS Open/Close.Form . . . . . . . . . . . . 143

Appendix B: Semi-structured Interview Schedule . . . . . . . . . 144

Appendix C: Letter of Invitation. . . . . . . . . . . . . . . . 145

Appendix D: Consent Form . . . . . . . . . . . . . . . . 147 


\begin{abstract}
Psychiatric case registers (PCRs) have proven to be a valuable tool in mental health services evaluation and research. Manitoba's Mental Health Management Information System (MHMIS) contains the information necessary to serve as a PCR. However, the quality of MHMIS data has not been assessed. Therefore, the objective of this study was to assess the reliability of the MHMIS data. Specifically, the objective was to determine the accuracy of information contained in the MHMIS as compared to client charts and to determine which factors influence completeness and accuracy.

The first part of this study involved a comparison of MHMIS information with client charts. Data on diagnosis, open date and close date, demographic information and contact information was obtained from client charts. Chart information was then compared to the same information extracted from MHMIS.

The second component of the study involved interviews with those who contribute information to MHMIS. Semistructured interviews allow estimation of sources of bias in both sets of data, explain differences between data sources and may facilitate measures to improve data quality. Questions were asked regarding understanding of the uses of MHMIS, beliefs about data accuracy, what factors are believed to influence data accuracy and the magnitude of influence. Information on required demographic variables, primary diagnosis, and
\end{abstract}


open and close dates are highly similar in the two sources of data. The correlation between data sources on the number of client contacts is also good. Agreement was highest for inpatient services. No differences in agreement were found based on type of clinician or region/facility.

This study establishes the reliability of MHMIS data and therefore facilitates use of MHMIS data for research. MHMIS data, in combination with hospital abstracts and physician claims data in the Manitoba Health Research Database, provides the information needed serve as a PCR and can be used for psychiatric epidemiology as well as for planning, monitoring and evaluating mental health services. 


\section{ACKNOWLEDGEMENTS}

I would like to thank my family, Gordon, Pamela, Brendon and Rebecca for their patience and understanding during the course of this research. Your love and support forms the basis for this achievement.

I would like to thank my committee for their guidance and support in this endeavour. My committee members Dr. Cameron Mustard, Dr. Leslie Roos and Dr. Barry Trute, and in particular my advisor, Dr. Douglas Tataryn, who were always there to answer questions while simultaneously fostering independent learning.

I would also like to thank all the individuals who facilitated this research either by participating in the interviews or by arranging access to information. In particular I would like to thank Mr. Chris Hauch for his assistance in guiding this project through the bureaucracy at Manitoba Health.

And finally, I would like to acknowledge the Manitoba Centre for Health Policy and Evaluation and the National Health Research Development Program for their Fellowship Awards, which allowed me to complete my Masters degree.

This research was supported in part by the National Health Research Development Program through a National Health Fellowship to Jean Renée Robinson. 
vii

\section{LIST OF TABLES}

Table 1 Agreement between MHMIS and client charts on demographic variables

Table 2 Source of demographic information abstracted from client charts

Table 3 Agreement between MHMIS and client charts on ICD-9-CM psychiatric diagnosis at the five digit level

Table 4 Agreement between MHMIS and client charts on ICD-9-CM psychiatric diagnosis at the three digit level

Table 5 Source of disagreement between MHMIS and client charts on primary diagnosis

Table 6 Agreement between MHMIS and client charts using hierarchical mental health categories

Table 7 Agreement between MHMIS and client charts on client contacts by clinician type and region/facility of service

Table 8 Correlation between client contacts identified by MHMIS and client charts

Table 9 Proximity of MHMIS open dates and close dates to client chart information on open and close

Table 10 Agreement between MHMIS and client charts on open and close dates 
viii

Table 11 Impact of service type, region/facility and clinician type on overall differences in agreement between MHMIS and client charts

Table 12 Overall agreement between MHMIS and client charts based on type of service 


\section{LIST OF ABBREVIATIONS}

DSM-III-R Diagnostic and Statistical Manual of mental disorders, third edition, Revised

DSM-IV Diagnostic and Statistical Manual of mental disorders, fourth edition

GLM General Linear Modelling

ICD-9-CM International Classification of Diseases, 9th edition, Clinical Modification

MHMIS Manitoba Mental Health Management Information System

PCR Psychiatric Case Register

PHIN Personal Health Insurance Number 


\section{INTRODUCTION}

The budget for provincial mental health services in Manitoba for the 1993/94 fiscal year exceeded 44 million dollars (Province of Manitoba, 1994). This total did not include expenditures for in-patient treatment at acute general hospitals, fee-for-service payments to psychiatrists and physicians for treatment of persons with mental health disorders or other costs, such as social services benefits to mental health clients. Despite the magnitude of expenditure, minimal research has been conducted on the use of mental health services in Manitoba or elsewhere. Recently, the necessity for evaluation of existing and proposed mental health services and programs has become increasingly apparent to health policy decision-makers, due to constrained budgets and changes in the delivery system. Research using administrative data has increasingly been used to provide substantive and timely information to inform policy decisions (Romano \& Luft, 1992). This methodology can also be applied in the mental health sphere.

In 1983, World Health Organization suggested that a Psychiatric Case Register (PCR) be defined as a "patient-centred longitudinal record of contacts with a defined set of psychiatric services originating from a defined population" (ten Horn, 1986 p. 170). PCRs are used to explore "disease frequency, course and outcome, causal associations and response to treatment and with the evaluation of services and therapies" (Fryers, 1987, p. 337). PCRs also provide 
information for planning, monitoring and evaluation of mental health services and can provide a sampling frame and controls for research.

Manitoba's Mental Health Management Information System (MHMIS) contains the information necessary to serve as a PCR. Collection of longitudinal information is possible due to unique personal identifiers; files can be built on individual contacts with any provincial mental health service. In addition to providing data on use of provincial mental health services, MHMIS information can be linked to information on services received for mental health problems from physicians and acute care hospitals. Coverage is virtually universal since the entire population of the province is eligible to receive provincial mental health services at no direct cost.

The Manitoba Centre for Health Policy and Evaluation has repeatedly assessed Manitoba Health data and found the data to be generally reliable (Roos, Montgomery \& Roos, 1987). However, the quality of data collected by MHMIS may be quite different as conditions under which data are collected differ substantially. For instance, Manitoba Health data is comprised of claims from fee-for-service physicians while MHMIS information is submitted by nonphysicians and is not the basis of remuneration.

Use of administrative data for research is dependent on accurate information. Administrative data was not collected for research and the necessary 
degree of rigor may not be present (Roos, Nicol, Johnson \& Roos, 1979; Bigelow, 1989). As a result, assessment of data quality should precede use of the data for research.

Therefore, the objective of this study was to assess the reliability of the MHMIS data by adapting methods developed by the Manitoba Centre for Health Policy and Evaluation in its assessment of the strength of the Manitoba Health Data Base. Specifically, the objective was to determine the accuracy of information contained in the MHMIS as compared to client charts and to determine which factors influence completeness and accuracy. Estimation of the degree and direction of any bias in the data will also be addressed.

\section{REVIEW OF THE LITERATURE}

\section{Applications of Mental Health Information Systems}

Computerized information systems are increasingly common for a variety of reasons including increased size of service organizations, a need for accountability to funding organizations, an emphasis on outcomes and mandated planning and evaluation (Tefft, 1983). Ideally, a mental health information system should meet the needs of a variety of users such as clinicians, administrators and researchers (Miller \& Willer, no date). While the needs of each group are different, the needs are not necessarily in conflict.

Clinicians are sometimes suspicious of the uses of administrative data and 
believe the information will be used against them. In fact, "All organizational levels perceive serious threats from computing, most often in terms of power and control to other levels, even though none are confident of gaining power" (Tefft, 1983 p 50). However, Binner (1993 p 54-55) suggests that

The power of information systems is based on the accuracy and relevance of the information they can produce. If they can maintain their integrity, they can serve as a countervailing force to the corrupting influence of power based on money, position, politics or other factors which can influence decisions within the mental health system.

Administrative uses of mental health information can result in streamlined services and improved client care through access to information on treatment history (Chapman \& Ostye, 1987); administrative data can provide information on which community mental health services can be planned and evaluated (Wing, 1983, Beecham, Knapp \& Fenyo, 1991). Administrative data has been used to examine the impact of intensive community services upon chronic hospital utilization (Bigelow, 1991), to examine patterns in the delivery of psychiatric care in Saskatchewan (D'Arcy, 1976, 1977a, 1977b) and Manitoba (Tataryn, Mustard \& Derksen, 1994) and to describe characteristics and health services utilization of alcohol and drug dependent individuals (D'Arcy \& Bold, 1983). Other research uses of administrative data include studies on the incidence and 
prevalence of mental disorder, studies of treatment course and outcome, sampling patients for clinical or epidemiological studies and studies of risk factors for mental illness (Munk-Jergensen, Kastrup \& Mortensen, 1993, Mortensen, 1995). One of the major advantages is the potential to follow up large cohorts over time. At present, few psychiatric case registers covering large populations exist around the world (ten Horn, Geil, Gulbinat, 1986). There are also few mental health information systems covering large populations which could be used for epidemiological research.

\section{Mental Health Information Systems and Case Registers in Canada}

Despite a universal health care system in Canada, there are few Psychiatric Case Registers; few provinces have mental health information systems which could serve as a PCR. In order to serve as a PCR, the information system must 1) be able to track individuals over time, 2) be linkable to information on mental health services provided by acute care hospitals and primary care physicians and 3) accurately capture information on clients and services.

Kingston, Ontario has a PCR which contains information on inpatient and outpatient services for two acute care hospitals and one psychiatric hospital (Woogh, 1987, 1988). A similar register exists in Thunder Bay, Ontario.

Ontario administrative information does not contain a personal identifier 
and tracking individuals over time is not possible, even in the same service. For instance, five hospital separation abstracts could represent five individuals or admission of the same individual five times.

Alberta mental health administrative data has been used for research although the primary use is management and planning. Information on services provided by the provincial Department of Health is captured but information on services provided by acute care hospitals and through outpatient services is not captured. Mental health information can not be linked to information on physician services. Inability to link information on mental health services with information on hospital and physician services presents problems since physicians outside the mental health system may treat six times the number of clients served by mental health services (D'Arcy, 1976).

Administrative information in Alberta is currently undergoing significant change. Responsibility for information systems (in health and mental health) has been devolved to a Provincial Health Board which will turn over responsibility for the system to Regional Health Authorities by March, 1997. Each region has been asked to develop an information system which includes mental health. The Provincial Health Board is also doing substantial development. The intent is that the Board will develop a uniform minimum data set with central linkage. The extent to which information systems developed by the Regional Health Authorities 
are compatible remain to be seen (R. Bland, personal communication, July 9 , 1996)

Saskatchewan collects information on mental health services provided to inpatients, outpatients and community services clients. This information is linkable to hospital and physician claims data. The mental health information system has been used for research since inception. In fact, research was part of the incentive to develop the information system.

Responsibility for mental health services in Saskatchewan was transferred to District Health Boards as of April 1, 1995 with only a few core services retained by the province. Although the Transfer Agreement requires continued reporting of mental health information, the amount of information submitted and the completeness of submitted information appears to have decreased (M. Stang, personal communication, July 8, 1996). Saskatchewan Health is currently investigating the extent to which information is incomplete. British Columbia has a mental health client information system initiated in 1987 which contains information on outpatient clients at approximately 100 mental health centres and both inpatient mental health facilities. Clients have a unique identifier which allows linkage of this information with other provincial health information. Clinicians submit information quarterly on each client. Information is not maintained on the numbers of client contacts or time spent with clients. The 
system is used for planning and administration. With the move toward regional governance, responsibility for information systems will rest with the regions.

Manitoba's MHMIS contains information on inpatient, outpatient and community services provided through provincial mental health programs. MHMIS information can be linked to hospital and medical services for these clients by a unique personal health insurance number (PHIN). Manitoba is in the preliminary phases of regionalization and the impact that regionalization will have is not known.

\section{Strengths and Limitations of Administrative Data}

The increasing availability of routinely collected data offers the opportunity for inexpensively monitoring the outcomes of different providers and institutions (Roos, Cageorge, Austin \& Lohr, 1985). Administrative data is particularly suited to studying concrete procedures like death and hospitalization (Roos \& Roos, 1989). Different data fields differ in reliability. Roos, Nicol and Roos (1983), in examining Manitoba Health Data, found identifying and demographic information to be of high quality, although address and marital status tend to become outdated. Discharge status was also found to be of high quality with procedure information next in quality and diagnosis presenting the most problems (Roos et al., 1982).

Measurement of health status using administrative data is difficult. 
Measures of health status have been developed which are significantly associated with other health measures (Mossey \& Roos, 1987) but some authors suggest that health care use should not be used to assess health status (Bergner, 1985). Patrick, Bush and Chen (1973) suggest that health status measures need to include information on functional status and prognosis in order to define health. This information is typically not available in administrative data.

\section{Reliability Studies}

Accurate data is of central importance in using administrative data to inform health policy or for research. The accuracy of each specific data element should be undertaken prior to large-scale research (Roos et al., 1979). Improvements in data collection can only be achieved if key problems can be identified and the frequency of occurrence assessed.

Reliability refers to the degree to which the results obtained by a measurement can be reproduced (Carmines \& Zeller, 1979; Campbell \& Fiske, 1959). Reliability may be defined as

The degree of stability exhibited when a measure is repeated under identical conditions. Reliability refers to the degree to which the results obtained by a measurement procedure can be replicated (Last, 1988, p. 114).

One method which can be used to check the reliability of administrative 
data is to compare information recorded in separate locations at a similar point in time. "Information recorded independently by separate individuals or organizations at two different times, or in two different data files is one key to performing reliability studies inexpensively" (Roos, Roos, Cageorge \& Nicol, 1982, p. 275).

Chart data can differ substantially from claims submitted for insurance purposes. Studney \& Hakistan (1981) found only $40 \%$ of physician claims submitted to the British Columbia Medical Services Plan were similar to chart data. Information in the psychiatric sphere was most likely to be inaccurate. Schwartz, Perlman, Martin, Schmidt \& Thornton, (1980) found substantial discrepancies in Medicaid billing for mental disorder which could be construed as a consistent pattern of distortion. Mental disorder continues to be a highly stigmatized condition (Klerman, Olfson, Leon \& Weissman, 1992).

Maximum reliability may be expected when data is unambiguous and not subject to professional disagreement. Since psychiatric diagnoses are ambiguous and subject to disagreement, they are likely to pose problems of reliability (Roos, Nicol \& Roos, 1983). It has been demonstrated that substantial changes in diagnosis can occur between initiation and termination of care, particularly for diagnoses such as depression and adjustment disorder (Snyder \& Strain, 1990). American hospitals have been shown to over-diagnose schizophrenia with chart 
confirmation of diagnosis in only $18 \%$ of cases studied (Lipton \& Simon, 1985).

In addition to inaccurate classification of clients, both the validity of the DSM-III classification system and the reliability of diagnoses in non-research settings have been questioned. Kirk and Kutchins (1992) suggest that agreement is poor between highly trained professionals using structured interviews and broad diagnostic categories. Reliability in clinical settings or for specific diagnoses will be even lower.

Collapsing diagnostic information into categories often increases the probability that a case will be categorized correctly, but categories are less sensitive and less meaningful than specific diagnoses (Mirowski \& Ross, 1989). Further, there is little agreement on the best way to group diagnoses (Sytema, Geil \& ten Horn, 1989).

\section{Factors Influencing Data Quality}

Workload and perceived importance of the data are likely to impact on the quality of data collected. Completion of forms has little impact on the work of mental health workers and the submission of information is often seen as an addon task (Nutter, 1983). Hence, motivation to comply with completion is minimal. The degree of similarity between insurance claims and chart data has been found to be negatively associated with workload (Studney \& Hakistan, 1981).

Some misdiagnosis is inadvertent, but deliberate misdiagnosis also occurs. 
In a survey of Registered Social Workers, Kirk and Kutchins (1988) found 87 percent of respondents were aware that less serious diagnosis than clinically warranted were used. Forty-four percent reported this occurs frequently. Respondents also reported Axis I diagnoses were assigned when not clinically warranted. Seventy-two percent were aware of use of more serious diagnoses to allow clients to qualify for reimbursement. "Over 80 percent indicate that thirdparty reimbursements often influence diagnoses" (Kirk \& Kutchins, 1988, p. 230). A national survey of psychiatrists in the United States also found that diagnostic information submitted to insurance companies was quite different than diagnoses provided in an anonymous survey (Sharfstein, Towery \& Milowe, 1980).

Human factors play a large role in mental health information systems (Fryers, 1987). Trute (1983) suggests that the consistency and accuracy of data gathering is fundamentally determined by personnel. Workers attach different values to data collection than researchers (U.S. Department of Health and Human Services, 1986). Practitioners see mental health information systems as a burden on their time and are concerned about the potential privacy risk to clients. These concerns may impact on the information submitted. Brown (1987) reported that mental health professionals make purposely hazy diagnoses to protect socially valued clients and will minimize and inflate diagnoses. Clinicians tend to make 
minimal non-troublesome diagnoses when forced to make a diagnosis, but insurers attempts to remove coverage results in more serious diagnoses being applied. A survey of social workers found that diagnosis is of little practical use to mental health practitioners. Ninety percent of respondents said the primary use of diagnosis was reimbursement. Only one third found diagnosis useful for treatment planning and one third felt diagnosis led to inappropriate treatment (Kutchins \& Kirk, 1988). Concerns about the impact of labelling clients may also impact on assigned diagnoses.

Information other than diagnosis may also be vulnerable to alteration. For instance, information such as the quantity of time spent with a client and the number of identified cases can also be influenced. For instance, in British Columbia, the reported time spent with clients rose substantially when it was suggested that numbers were low. Similarly, a memo about short-term assessment was anticipated to produce an increase in reports of short term assessment (B. Douglas, personal communication, February 2, 1995). Accurate information is required for administration as well as for research. However, data collected and used to administer a system may not be collected with the required degree of rigor for research (Roos et al., 1979).

Psychiatric Case Registers face some additional influences, such as inability to maintain and update demographic information (Fryers, 1987). 
Registration information on all Manitoba residents is maintained and updated on an ongoing basis. Current information can therefore be obtained for mental health clients even when the individual is not receiving services.

\section{Confidentiality}

The legal perception of the possible harm and unfairness of psychiatric diagnoses and mental health classifications, whether accurate or not, have created legal responses to mental health information systems which tend to be restrictive (Dickens, 1983). However, concerns about confidentiality are largely related to administrative rather than statistical uses (Trute \& Tonn, 1982). Wing (1986 p 360) suggests that "no single example of harm done to a person whose data have been entered in a research register has ever been recorded". Restrictions on use of mental health information can prevent research which has potential benefits. For instance, the Mannheim psychiatric case register was established to monitor movement of clients from institutions to the community, for long term epidemiology and to examine trends in rates of psychiatric disorder. The register was closed in 1981 because interpretation of laws precluded use of the information without written consent from all subjects. Aside from difficulty obtaining valid consent from clients suffering from mental illnesses such as dementia, clients could not consent to studies that had not yet been planned (Hafner \& Pfeifer-Kurda, 1986). Similar interpretations resulted in reduction in 
the quality and quantity of information in the Iceland Psychiatric Register (Helgason, 1986).

In 1984, the Parliament in England passed the Data Protection Act which seeks to protect privacy while allowing access for research (Wing, 1986b). The Mental Health Act in Manitoba (1987) balances client confidentiality and access by placing restrictions on access to mental health records but specifically allowing use of client records in duly authorized research.

\section{BACKGROUND}

\section{Manitoba Health Research Data Base}

The Manitoba Health Research Data Base includes a population based longitudinal registry and a number of separate data files. The data files include hospital admission/separation abstracts, physician services claims, personal care home and nursing home administrative records, Manitoba Immunization Monitoring System records for children, physician registration files, provincial drug insurance program and vital statistics files (Roos et al., 1993). MHMIS is another separate data file containing information on mental health services provided through mental health facilities and through regional community based services. All of these data files can be linked using a unique PHIN.

Information in the Research Data Base is administrative data collected by Manitoba Health. Manitoba Health makes the files available to the Manitoba 
Centre for Health Policy and Evaluation, which houses the Research Data Base, after the end of a fiscal year.

Registration data is relied on to provide a consistent, accurate source of demographic data and coverage information on all Manitoba residents. The population is universally covered and demographic data is reasonably current as it is maintained jointly with other health information. For example, address information is confirmed each time a client visits a physician. Information that a client has moved to a new address is submitted to Manitoba Health and confirmation is sought. All Manitoba residents are covered for health services under the Manitoba Health Services Insurance Plan and participation in the plan is virtually complete since residents are not required to pay any premiums. The registered population closely matches the provincial population as determined by the census. Marital status derived from the registration data is in agreement with vital statistics information at least 95 percent of the time (Roos et al., 1993).

Information on hospital and physician services contained in the Research Data Base has been repeatedly assessed and has been found to be relatively complete (Roos et al., 1987). Two important potential confounding factors, overreporting and underreporting are considered minimal: Since remuneration for services is contingent on submission of claims, underreporting is minimized. Overreporting is minimized by monitoring and investigation of outliers (Roos \& 
Roos, 1989). However, reliability of the data may change if certain factors, such as method of remuneration, are changed. For example, an informal study of a medical unit which changed from fee-for-service to salary found that compliance with submission of physician claims declined by 30 percent despite a requirement to continue submitting claims for record-keeping purposes (B. Postl, personal communication, Sept. 29, 1994).

\section{Provincial Mental Health Services}

Provincial mental health services in Manitoba are delivered through inpatient and outpatient services of mental health facilities and regional community based services. Brandon and Selkirk Mental Health Centers each provide treatment to Manitoba residents from their catchment area on an inpatient and outpatient basis. Inpatient services provide 24 hour residential care to clients while outpatient services provide care and support for these same clients on a nonresidential basis. Eden Mental Health Center also provides inpatient and outpatient services but differs from Brandon and Selkirk in that it is a regional rather than a provincial facility.

Regional mental health services are provided by multidisciplinary teams located in each of the eight provincial health and social services regions. Services include community based prevention, rehabilitation and support for mental health clients. 
The mandate of Manitoba's mental health services is to "respond to the needs of individual Manitobans who face mental distress and aim to assist those individuals in maintaining or restoring their ability to function with responsibility, independence and health" (Manitoba Health, 1992, p. 3). Mental health services include assessment, treatment, outreach and emergency services. Individuals can refer themselves for services or referrals can be made by physicians, community agencies and facilities. However, access to inpatient services is controlled by physicians/psychiatrists. In practice, the focus is on direct service to acutely and persistently mentally ill clients and duplication of other services is avoided. For instance, counselling for substance abuse is handled by the Addictions Foundation and these services are not provided by mental health services.

\section{MHMIS Data}

Manitoba's MHMIS contains information on clients served by Provincial Mental Health Services in mental health facilities or in community based regional services. The information includes demographic information, dates for opening and closing the case, referral source, diagnosis, therapies provided, case manager and so forth. In addition, each time a clinician has contact with a client, information is collected on the date, type of contact (e.g. in person or by telephone), and services provided. Thus, MHMIS contains comprehensive information on mental health services provided to Manitoba residents. 
The information contained in MHMIS is not homogenous. Different methods are used to collect information in each type of service (inpatient, outpatient and regional services) and the information collected also differs. For instance, MHMIS information submitted by regional services is collected and coded by the clinician and entered by clerical staff. MHMIS information on inpatients at mental health facilities is abstracted from client charts, coded and entered by clinical records staff. Regional clinicians contribute information on client contacts, while this information is not submitted by clinicians in inpatient services.

Even within a single mental health facility, the process of information collection and type of information submitted can vary. In outpatient departments, the senior secretary abstracts information from the client chart, codes and submits information for the MHMIS Open/Close form (Appendix A), while contact information is submitted by the clinician. Regional clinicians submit information on client contacts while only partial contact information is collected for inpatients. As a result of different information contained and different methods of data collection, separate assessments were required for inpatient and outpatient services of mental health facilities and for regional services.

Data quality may also vary based on the region or facility where service is provided and by type of clinician (physician, psychiatric nurse, social worker 
or other clinician). Each of the eight health and social service regions has its own unique characteristics as do the three mental health facilities. Different types of clinician within a region may have different degrees of familiarity with diagnostic classification and different attitudes toward diagnosis. Clinician types vary with setting. For instance, information from the Mental Health Division suggests that regional services staff are primarily registered psychiatric nurses (RPN) and social workers. Diagnoses made by these individuals may differ from diagnoses made in mental health facilities where diagnosis is typically made by a team headed by a psychiatrist. Therefore, data analysis is conducted by region/facility and clinician type.

Not all information fields in MHMIS require assessment. There are 98 fields in the MHMIS Open/Close form and an additional 19 fields on the Encounter forms. This total does not include additional fields found on the Case Pending or Change of Status forms. Not all fields contain required information and not all fields are essential for research. For example, completed by and entered by fields and their corresponding dates are of little importance in research on client characteristics, patterns of care or outcomes.

Information which is important for research can be grouped into four categories. These categories include 1) demographic information, 2) diagnosis, 3) contact information and 4) open date and close date. Demographic information 
includes gender, date of birth, marital status and postal code. Highest level of education achieved, employment status, occupation, treaty status and religion are optional fields which provide important demographic data for research. A case is open when service delivery is initiated and closed when service delivery has been terminated. The quality of data in each category may differ. For instance, based on preliminary interviews which were held with individuals contributing information to the system, demographic data was considered to be of high quality with contact information of medium quality and therapies information was considered poor.

The term client is preferred to describe a consumer of mental health services. However, consumers receiving treatment at a mental health facility have historically been referred to as patients. This view is still reflected in the categorization of services by the facilities as inpatient or outpatient services. For consistency with existing terminology, the services are referred to as inpatient or outpatient services but consumers are referred to as clients.

\section{METHODS}

\section{Overview of Methods}

The objectives of this study were to 1) determine the reliability of information contained in MHMIS, and 2) determine which factors influence completeness and accuracy of MHMIS data as well as estimate the degree and 
direction of any bias in the data. Separate methods were necessary to examine each of these areas.

Congruence between administrative data contained in MHMIS and client chart data was determined by conducting chart reviews. Current demographic information and diagnosis, all contacts with clients during the study period and the date the case was opened and closed were abstracted from client charts and compared to the same information in MHMIS. Contact information was not abstracted from inpatient charts since mental health facilities submit contact information for some clinicians but not for others. For each type of data, chart information was then compared to MHMIS information for the same client.

Comparison of client charts to MHMIS information demonstrates the reliability of MHMIS data and the extent to which MHMIS data could be used as a substitute for chart data in conducting research. However, chart reviews cannot identify the extent to which the findings accurately represent reality or suggest how improvements in data quality can be obtained. In order to identify factors which influence both sets of data, to explain differences between data sources and to facilitate identification of measures to improve data quality, semistructured interviews were conducted with individuals contributing information to MHMIS.

This methodology does not allow for examination of the validity of data contained in either charts or MHMIS. Validity is a measure of the degree to 
which an instrument measures what it is supposed to measure (Last, 1988). Agreement between MHMIS and client charts on diagnosis does not necessarily mean that the diagnosis is accurate. Determining the validity of MHMIS data would require a gold standard against which the data could be tested. Since there is no readily available gold standard for a given clients demographic information, diagnosis, contacts, or open and close date, this study focuses on the reliability of the MHMIS information as compared to client charts.

The study was initially conducted in three regions and two mental health facilities. Data from these region/facilities was used to conduct a generalizability analysis. The purpose of the generalizability analysis was to determine the extent to which additional information would be obtained by collecting data from all eight regions and three mental health facilities.

\section{Population}

\section{Chart Reviews}

The target population for chart reviews included all clients served through provincial mental health programs in Manitoba. This included clients served by regional mental health services in any of the eight provincial health and social service regions and clients served through inpatient or outpatient services at mental health facilities in Brandon, Eden or Selkirk. Clients who received mental health services in Acute Care Hospitals, through programs run by nongovernment 
organizations (such as Crisis Stabilization or Klinic) and clients of Psychealth at the Health Sciences Center were excluded since these programs do not contribute information to MHMIS. Psychealth contributed information to MHMIS on a limited basis for a short period of time but has developed its own information system and is therefore excluded.

Figures from Manitoba Health (1994) indicate that mental health facilities in Manitoba served about 1,500 inpatients in fiscal year 1993-94 with an average daily census of about 520 individuals. The active client count for outpatient services of mental health facilities averages approximately 3,000 clients. Regional services saw about 7,650 clients during fiscal year 1993-94 with an average active client count of about 4,600 .

\section{Interviews}

The target population for interviews was dependent on the type of service. The population included those individuals responsible for coding and submitting information for entry into MHMIS. For regional services this included all direct service clinicians (Community Mental Health Workers), regardless of whether they worked part-time or full-time. For inpatient services, the target population included clinical records staff who abstract and code information from charts. To explore factors influencing outpatient services, both direct service providers and clerical staff responsible for coding were included in the population. 
There are approximately 160 Community Mental Health Workers (CMHW) employed by Manitoba Health. About 60 percent of CMHW are RPNs and 40 percent are social workers. There are only a few individuals with other preparation such as Registered Nurses or Occupational Therapists working as CMHW. Outpatient services staff number approximately 60 and include physicians and psychologists as well as RPNs and social workers. There are about 35 clinical records or clerical staff responsible for abstracting and coding inpatient information.

\section{Sample}

\section{Chart Reviews}

The computerized list of all MHMIS cases contained in the Manitoba Health Research Database was used as the sampling frame for client charts. The population eligible for selection included all cases which were open on November 1, 1994 and any cases opened from November 1, 1994 to April 30, 1995. This sampling strategy made it possible to review a cross section of cases including cases that are open and cases that are closed. In total, 12,773 cases were eligible for entry into the study. The sample was stratified by type of service and cases were selected randomly within each region/facility. A total of 30 cases were selected for each region/facility to ensure that 20 cases could be reviewed. For instance, if certain client charts in the first 20 were not available, another client 
chart could be reviewed.

Cases were selected using a SAS macro for random sampling of datasets developed by Charles Burchill at the Manitoba Centre for Health Policy and Evaluation. The macro assigns a random number to each case and sorts cases by the random number within each type of service and region/facility. This randomization strategy provided a prioritized list of client charts in each region/facility for review, allowing review of additional cases if required.

A single client was eligible for selection more than once if they received treatment from more than one type of service or if they had more than one episode of care during the study period. For instance, a client receiving treatment from regional services and inpatient services would have separate MHMIS records for each episode of care and separate client charts in each service. Selection of the same client more than once would not influence the results since each case was reviewed separately. Inpatient cases were reviewed using inpatient charts and regional cases were reviewed using regional charts. Only information from the selected episode of care was reviewed.

\section{Interviews}

The sampling frame for clinician interviews included clinicians identified as the case manager for sampled client charts. The same macro and the same process used for selection of client charts was used for selection of clinicians for 
interview. A random sample of clinicians was selected from the sampled cases using the clinician number for the identified case manager. Random number generation and sorting by the random number yielded a prioritized list of five clinicians so that three individuals in each region or outpatient setting could be interviewed. If the same clinician appeared more than once in the first three places, or if a clinician declined the interview, the next individual on the list could be approached.

It was anticipated that selection of the clinical records and clerical staff would be conducted in the same manner using the entered by field in MHMIS. Although this field is required, the information is not entered into MHMIS and could not be used for sampling. Discussion with clinical records staff in two facilities revealed that there are a small number of individuals who are involved in submission of information to MHMIS and, since they may have specialized roles, a specific set of individuals should be interviewed. At least one individual in each role was identified for interview. In one facility, all clinical records or clerical staff having responsibility for MHMIS were selected.

In total, 280 charts were selected for review with 20 client charts for each region/facility and type of service. This represents 2.3 percent of all eligible cases. Twenty four CMHW (15 percent of all regional services staff), nine outpatient staff ( 15 percent of all outpatient staff) and nine clinical records staff 
(26 percent of all clinical records staff) were selected for interview with additional names to ensure this number could be interviewed. A total of 42 persons were selected for interview. The sample size was chosen by balancing a desire for maximum information with a manageable number of charts and interviews.

\section{Instrumentation}

\section{Chart Reviews}

Information for each MHMIS data field is obtained and recorded in a different way. For instance, demographic information is collected once from the client at the time a case is opened while contact information is generated by the clinician on an ongoing basis. Although MHMIS and chart data are compared and differences based on type of service, clinician type or region/facility are explored for each variable, analysis of each data field differs based on the unique characteristics of the variable. A detailed description of each variable and explanation of how the variable is analyzed is provided along with the results for that variable. Discussion here is limited to general information that applies to all data fields.

Demographic information, diagnosis and open and close date are captured on the MHMIS Open/Close form. This form is maintained on client charts and is used by some clinicians for reference. Information may be stored on this form 
that is not documented elsewhere on the client chart. In conducting the chart reviews, information was obtained from the MHMIS Open/Close form only if the form was the only available source of information. The extent to which information from the MHMIS form was used for comparison has been identified for each affected analysis.

Manitoba Health data, including MHMIS, is extracted and made available to the Manitoba Health Research Data Base once a year. Data is extracted in June, about three months after the end of the fiscal year, to ensure that data for the fiscal year is relatively complete.

\section{Interviews}

A semistructured interview schedule was developed to guide the interviews. The schedule was developed on the basis of a review of the literature and the preliminary interviews. The interview schedule was piloted with three CMHW and necessary revisions were made (see Appendix B).

\section{Implementation}

Approval to investigate the feasibility of a study was granted in July of 1993 by Mr. Reg Toews, then Assistant Deputy Minister of Health. Following discussions with Mr. Chris Hauch, a Senior Clinical Consultant at the Mental Health Division with responsibility for MHMIS, preliminary interviews were held. A total of 12 individuals from four regions and two mental health facilities 
were interviewed. A proposal was developed and was approved by the thesis committee on March 23, 1994.

A number of approvals were required in order to conduct this research. In addition to approval from the Faculty Committee on the Use of Human Subjects in Research, support for the project was necessary from the Mental Health Division of Manitoba Health and the Manitoba Health Access and Confidentiality Committee. Under the Mental Health Act, approval was also required from the Medical Officer in Charge of each mental health facility. Since Selkirk Mental Health Center has a separate Ethics Committee, approval from this committee was required prior to approval of the Medical Officer. In keeping with the spirit of the legislation, approval was also sought from the Regional Director in each of Manitoba's eight Health and Social Services areas. A total of 14 approvals for the study were required and received.

All analyses data were conducted using MHMIS data contained in the Manitoba Health Research Database. Prior to receipt of MHMIS data by the Manitoba Health Research Database, all personal identifiers such as name and address are removed and the PHIN is scrambled by Manitoba Health. In order to protect client confidentiality in this study, client identifiers such as name or PHIN were not used. An identifying number and a rather circuitous process was used.

A sample of MHMIS clients was obtained from MHMIS data stored in the 
Research Database. The scrambled PHIN and identifying number were supplied to Manitoba Health. The scrambled PHIN was then converted to the real PHIN by Manitoba Health staff and both numbers (real PHIN and identifying number) were supplied to regional or facility staff. When the charts were provided for review, the identifying number for the case was also provided. Only the identifying number was retained on data collection sheets. In this way the researcher was never in possession of personal client identifiers and all identifying information remained in the possession of Manitoba Health.

Considerable differences between regions were anticipated as regions differ in several important ways (Tataryn, Roos, \& Black, 1994; Tataryn, Mustard, \& Derksen, 1994). Since maximally different regions were needed for the generalizability analysis, the regions selected included one southern urban region, one southern rural region and one northern region, in accordance with the cited literature.

Interviews and chart reviews for the generalizability analysis were conducted in three regions and two mental health facilities. Chart reviews and interviews related to inpatient and outpatient services were conducted on-site at each provincial mental health facility. Chart reviews and interviews related to regional services were conducted on site in the three regions. Since client charts and clinicians may be located in a variety of suboffices in a region, chart reviews 
and/or interviews took place in 13 region/facilities. Interviews took about one hour to complete.

\section{Analysis}

\section{Chart Reviews}

Information from the chart reviews was entered into a SAS data set. All statistical analysis were conducted using SAS version 6.09 for Unix.

The original plan for analysis involved using Kappa as the measure of agreement. Kappa is considered to be an ideal method for assessing agreement between categorical variables because it uses the marginal proportions to provide an error corrected measure of agreement (Spitzer, Cohen, Fleiss, \& Endicott, 1967; Cohen, 1968; Fleiss, 1981). However, a number of obstacles to the use of kappa became apparent including: 1) Information is usually found on the client chart, in MHMIS or on both data sets. The situation where information is missing on both data sets does not occur except for a few optional demographic variables and therefore this cell is usually empty. Kappa values are negative in a situation where one of the cells has no information. 2) Agreement between data sources on at least some data fields was expected to be between 0.7 and 0.8 . A simulation conducted in collaboration with D. J. Tataryn (advisor) demonstrated that the confidence limits of kappa are highly variable and can be expected to be quite large at a kappa of 0.7 or 0.8 . 3) Although kappa can be 
used for a small number of categories, it is ideally suited to dichotomous variables. Information such as birth date would have to be collapsed into as few as six categories for assessment of agreement. Similarly, diagnosis would have to be collapsed into a small number of categories. Agreement on such as small number of categories would not be meaningful.

The rationale for using kappa was largely based on the ability to correct for chance. It is highly unlikely that the open date for a specific case according to the client chart would match the MHMIS open date by chance. Similarly, it is unlikely that the diagnosis identified in client charts would match the MHMIS diagnosis at either the five or three digit level by chance. Therefore, simple agreement between the two data sources is considered to be a fair, and even stringent, test of agreement.

Agreement on demographic data was assessed for each required and optional data field and for the required demographic data as a whole. Agreement for diagnosis was assessed using agreement at the three and five digit level as well as agreement within a larger diagnostic class. Open and close dates were assessed separately and as a group. Following separate assessment of each type of data, overall agreement was assessed. Differences between types of service, clinician types and region/facilities were explored for each type of data and for the overall data using General Linear Modelling (GLM). 
General Linear Modelling combines regression and analysis of variance to statistically separate the effects of several factors on an outcome and determine which, if any of the factors are relevant to the outcome. GLM was used in this study to determine whether clinician type, service type, region/facility or interaction between these variables was related to each variable separately. GLM is also used to model the influence of these explanations on the information as a whole.

\section{Interviews}

Interview tapes were reviewed, issues raised were identified and key segments were transcribed. The data was aggregated into themes. Where a particular view or issue was related to a single service type, clinician type or region/facility, this information was also noted.

Results from the interviews and comparison of chart and MHMIS data were reviewed to identify which factors are believed to influence data accuracy and influence use of MHMIS data for research.

\section{Ethics}

Subject confidentiality was protected by obtaining informed consent from individuals who were interviewed, by using identifying numbers rather than personal identifiers such as name or PHIN for chart reviews and by maintaining information in a secure location. Information is reported in aggregate only, so 
that neither individual clinicians nor clients can be identified.

Clinicians and outpatient staff were contacted individually to set up interviews. A letter was sent to provide information about the study and what was being requested as well as to invite their participation (Appendix C) . A follow up phone call was made to answer any questions and to set up an appointment. Clinicians were offered the opportunity to be interviewed at work or away from the work setting if preferred. In order to ensure that clinicians felt free to express their views, regional managers were not informed which individuals would be interviewed.

Individuals who were interviewed all signed a consent form (Appendix D) which again provided information on the purpose of the study, what was expected of them, any risks or benefits. Each individual had full opportunity to give informed consent to the interview. Individuals were informed that neither participation nor refusal to participate in the study would be communicated to their employer.

Numbers rather than names were used on all interview tapes. Interview tapes and information sheets are kept under double lock in a secure location as is standard protocol in all mental health service agencies. The consent forms and lists linking clinicians to the tapes are kept in a separate locked location.

The Mental Health Act of 1987, Section 26.9 (3) states that 
The medical officer in charge of a psychiatric facility in which a clinical record is prepared and maintained may disclose or transmit the record to, or permit the examination thereof by ...

(g) any person for the purpose of research, academic pursuit or the compilation of statistical data where the person agrees in writing not to disclose the name or other means of identification of the patient and not to use or communicate the information for any other purpose.

Since legislation allows use of mental health data for research, individual client consent is not required. However, a signed oath of confidentiality was provided to Manitoba Health and to each region/facility requesting one.

Client data in regional service charts is not covered under existing mental health legislation. In keeping with the intent of the legislation, access was requested through the Regional Director in each region. Clients are advised on entry to service that their records may be used for statistical purposes or for research and that they will not be identifiable in any reports.

\section{Generalizability Analysis}

Generalizability analysis is based on Generalizability theory, also known as G-theory. G-theory uses analysis of variance to partition sources of variation and identifies the relative influence of variables such as region/facility, type of 
service and clinician type on the generalizability of agreement between MHMIS and client charts. Information on the variability of each element is used to provide information on the extent to which the results would change if more information was available. "The concept of reliability, so fundamental to CT [classical theory], is replaced by the broader and more flexible notion of generalizability" (Shavelson, Webb \& Rowley, 1989 p 922). G-theory does not ask about how closely the results reflect real scores, but subsumes reliability into a question of generalizability of the results.

Information on the extent to which variability of a specific facet (source of error) would be reduced by adding more information is particularly useful for deciding where resources for a study are best invested. For instance, if variation based on region/facility is small and variation between clinician types is large, the study can be maximized by increasing the number of clinician types. Very little new information would be obtained by adding information from more region/facilities. In this study, the number of service types and the number of clinician types can not be changed because all conditions of the facet have already been included. The generalizability study was used here to determine whether reviewing client charts in all regions and facilities would produce substantial change in the outcome. Due to the cost of travel to the additional region/facilities and the time involved in data collection, it was important to know that substantial 
new information would be obtained.

\section{Detailed Methods}

A single measure of agreement between MHMIS and client charts was needed to conduct the generalizability analysis. The measure was constructed using all four categories of information found on both data sets, namely diagnosis, contact information, demographic data and open and close dates. For the purposes of this analysis, diagnosis refers to primary diagnosis and demographic data refers to the required demographic variables, namely date of birth, gender, marital status and postal code. Agreement on open and close dates refers to agreement on dates within one week. Each category was given the same importance, as were variables within each category. Specifically, each category was assigned a value between zero and one based on agreement for each possible item. For example, a case that agreed on three out of four demographic variables, six out of 12 contact dates, primary diagnosis and open date but not close date would be calculated as follows:

$$
(0.75+0.5+1.0+0.5) / 4=0.69
$$

The proportion of overall agreement for this case is 0.69 . Since contact information is not available for inpatient cases, the summary variable for inpatients excludes agreement on contacts. Using the above information, an inpatient case would be calculated as 


$$
(0.75+1.0+0.5) / 3=0.75
$$

Inpatient and outpatient services of a single facility are not independent from one another but regional services are independent. Although region/facility is only partially nested within service type, region/facility is considered to be nested within service type for this and subsequent analysis.

Variance estimates for the generalizability analysis were calculated using the SAS VARCOMP procedure. The MIVQUE procedure in SAS VARCOMP has no distributional assumptions and is capable of dealing with unbalanced designs (Bell, 1985).

\section{$\underline{\text { Results }}$}

The generalizability coefficients which were calculated were very small and hence were difficult to interpret. After extensive investigation of the issue, including contact with Dr. Noreen Webb who publishes in the area, it became apparent that generalizability analysis could not be used with this data. Separate information, preferably counts, from each observer (chart and MHMIS) would be required. In this study, information form MHMIS and client charts is collapsed into a single measure of agreement and separation of the two is not easily accomplished. Nesting of client within a region/facility and partial nesting of region/facility within service type, also posed a problem since the variance related to client could not be isolated. 
Although conducting a generalizability analysis was not possible, based on the results of GLM for each variable separately and for an aggregate measure, it appeared there would be minimal benefit to further data collection. GLM can be used because inclusion of variation based on rater or individual clients is not required; the model can be constructed using only region/facility, service type and clinician type.

Since additional data collection was not required, the remainder of the analyses were conducted using information from the three regions studied as well as information from inpatient and outpatient services of two mental health facilities.

\section{DETAILED METHODS AND RESULTS}

\section{Interviews}

Semistructured interviews were conducted with individuals who contribute information to MHMIS. These individuals included clinicians who code and submit information to MHMIS as well as clerical and clinical record staff who abstract and code information for submission. In total 22 individuals were interviewed including 15 clinicians and seven clinical records or clerical staff. The clinicians included two psychiatrists, nine RPNs and four social workers.

Only one individual refused to be interviewed. The individual is a physician who declined on the basis that he did not complete any MHMIS forms 
and had no knowledge the system. Individuals who were interviewed were asked to describe the flow of information and the points at which they had contact with MHMIS. They were asked about accuracy and completeness of MHMIS information as well as what factors influence accuracy and completeness. Finally, individuals were asked whether they use MHMIS information for any purpose.

\section{Flow of Information}

\section{Inpatient Services}

The flow of information, or the actual procedures by which MHMIS data forms are completed, for inpatients differs slightly between mental health facilities. In one facility clinical records staff use information from admission forms to fill out the MHMIS Open/Close form. Admission date and time serve as the open date and time. All required demographic information is contained on the admission forms. Optional demographic information not contained on the admission forms may be obtained from the nursing assessment or previous records.

A case is usually opened using a diagnosis of 7999 (not yet diagnosed). After a diagnostic conference has been held, the Health Records Technician provides the ICD-9-CM (International Classification of Diseases, 9th Revision, Clinical Modification) codes for the diagnoses that were assigned, even if the diagnosis is made using DSM-III-R (Diagnostic and Statistical Manual of Mental 
Disorders, Third edition, Revised). Once diagnoses are coded, the original 7999 diagnostic code in MHMIS is replaced.

Information on client contacts is submitted by some clinicians such as dietary, psychology and social work but medical staff and nursing staff do not generally submit information to MHMIS. Contact information is entered by clerical staff.

A single therapy code, 102a (diagnostic assessment) is used to open a case. On most units information on therapies provided is submitted by the unit on discharge and the additional codes are entered in MHMIS.

Within a day of discharge, cases are closed using limited information such as date of discharge and the unit the client was discharged from. Information from the discharge conference is typed and the discharge diagnosis is coded by the Health Record Technician prior to entry of this information. Since the case is already closed, therapy and referral information, discharge diagnosis and discharge time are entered as a clinical status change on the closed case. Cases are closed immediately after discharge even though all the information is not available in order for the case to be opened to outpatient services. Cases can be open to regional services and to a facility at the same time but can not be open to both inpatient and outpatient services at the same time.

The flow of information in the second facility is similar to the first except 
that cases are opened using the admission diagnosis rather than a general code such as 7999. If diagnosis is later changed, this information is entered as a clinical status change.

\section{Outpatient Services}

A variety of mechanisms exist to collect and enter information into MHMIS for outpatient services. In one facility the MHMIS Open/Close form is initiated by clerical staff using information from the intake/referral form. The case is opened using 7999 as a diagnosis and a copy of the partially completed sheet is given to the clinician. After the clinician has completed their assessment, the form is completed, diagnosis is entered on the form and it is returned to clerical staff for entry. Cases are usually opened as of the date a client is first seen. It is possible to open a case as pending case if information is incomplete, but this is not seen as useful and is rarely used. The date a case is closed is determined by the individual clinician.

Clinicians code all information on the form, including diagnosis. In some cases diagnosis is transferred directly from the discharge information since a new assessment is only required on clients who have not been seen in six months. Diagnoses made by clinicians who are not physicians are considered to be diagnostic impressions.

In the other facility, clerical staff collect initial information directly from 
the client and wait for a diagnosis from the clinician to open the case.

Contact sheets in both facilities are completed and coded by clinicians and are submitted to clerical staff for entry. However, medical staff (physicians/psychiatrists) have assigned secretaries that complete and code the contact information (including therapies) based on dictation.

\section{$\underline{\text { Regional Services }}$}

All MHMIS forms for regional clients, including the Open/Close form, Encounter form and Change of Status forms, are completed and coded by clinicians. Data is entered by clerical staff in the region.

\section{Accuracy of MHMIS Information}

\section{Demographic Information}

Demographic information was considered quite accurate by individuals participating in the interviews. Accuracy is limited on occasion by availability of the information and by reliability of the information source. For instance, accuracy depends on "Whether the client is actually giving you the real goods. You take it at face value, all of it. (RPN)"

The degree to which the information is complete depends on the ease of data collection. Clinical records staff identified that information which is not readily available on the client chart, for instance on the intake, admission or assessment forms, is not always entered. Some clerical staff seek out information 
from other documents or from clinicians to complete the forms but other clerical staff do not.

I would say it all depends on how keen a person [who fills out the information or enters the information] is to provide accurate reports. The amount of effort the person wants to invest may affect whether the information is correctly classified or if it is entered at all (Clinical Records).

Clinicians differ in the amount of optional information which is submitted. Some clinicians submit only the required information and others routinely complete all fields. Clinicians are more likely to record the optional information if the information is considered useful to them or is easily collected. Clinicians also differ in their views about what information is important to collect. A clinician who works with children might consider education to be important and would therefore collect information on education routinely; another clinician may consider religion or social allowance number to be important and would collect this information. Conversely, a variable such as treaty number may not be collected if it is not considered relevant to treatment.

Some clinicians find information on the MHMIS Open/Close form to be useful when looking for specific pieces of information. One clinician reported that the MHMIS face sheet is a working part of the client chart and is where she 
would look for information such as family doctor or education. "Actually it [MHMIS Open/Close form] is kind of a focal point for all those very concrete pieces of information". Other clinicians do not refer to the form. Several clinicians, particularly those who do not code their own information, identified that the MHMIS Open/Close form is not useful for reference because information such as occupation, marital status, referral source and so forth is coded. Coded information is less useful than text for quick reference.

Information on the MHMIS Open/Close form is often duplicated in the client assessment. Clinicians consider it important that the assessment contain complete information even if the information is documented elsewhere on the chart. Some clinicians record information either on the MHMIS form or on the chart to minimize duplicate recording.

Two clinicians identified that they complete the MHMIS Open/Close form (except diagnosis) with client as part of the intake process. Completion of the forms with the client was seen as a means to integrate completion of the forms into the work and minimize the burden of paperwork.

The first thing we do when we see a client is fill out the form with them. And maybe there just might be a couple of things that we don't do until they are gone like perhaps the diagnosis. That might not even be done for a couple of times until you get a clear picture. But I think because it is 
part of the intake process we do have sufficient time.... There is always time to do it properly [fill out the form] and to decide how it is going to be filled out.

\section{Diagnosis}

Only one of the clinicians interviewed considered diagnosis to be inaccurately captured in MHMIS but several other clinicians identified that the diagnosis (or diagnostic impression in the case of nonphysician clinicians) was not as specific as it could be. One psychiatrist identified that accuracy of the diagnosis would be influenced by:

The detail of our dictation and whether it corresponds to codes as they exist would be an important component. If we have an unclear diagnosis, if we have just simply 'schizophrenia' well there is many codes that go under schizophrenia depending on what type it is, whether it is chronic or an exacerbation or whatever. There is many different categories.

However the clinician also stressed that in the event the diagnosis is not clear the clinical records staff should approach the clinician for clarification and clinical records staff in that facility confirmed that this is the process.

In cases where diagnosis has been established prior to referral, the diagnosis is usually reviewed by the clinician. Clinicians identify that the diagnosis is usually accurate but if it appears to be inaccurate the client will be 
reassessed.

The precision of diagnoses may also be influenced by the requirement for a diagnosis to open a case. Clinicians are reluctant to provide a diagnosis on the basis of a single visit but can not open the case without a diagnosis and can not enter contact information until a case is open. Some clinicians delay opening the case until they have a diagnosis while others provide a diagnosis immediately and still others open with a general diagnosis and then change it.

Some of us tend to be more general and others tend to be more specific [in making a diagnosis] straightaway. Some of that is just a belief system I think. I am not necessarily willing to sit down and give someone an Axis I or Axis II diagnosis when I want several months to decide that for sure. And maybe that will get changed or maybe it will remain a very general [diagnosis].

One clinician reported that a diagnosis or at least a differential diagnosis is provided by the end of the first contact but a final diagnosis is not provided until one is made. One clinical records staff identified that they "hassle" the clinical staff to "get the diagnosis out" so that cases can be opened but other staff say this is rarely required.

Workload may also influence the level of detail in diagnosis and confidence in diagnosis. 
Workload and how much you've got going at the time is one of the biggest influences on how accurate the information is. Whether you're going to take that extra bit of time to really look at things when you're coming up with diagnosis specifically, for instance, or whether you're going to get this in. I think I would tend to be much more general [in diagnosis] if I had to get it [diagnosis] in.

Clinicians varied greatly in their view of the importance of diagnosis and comfort with making diagnoses. Some clinicians considered diagnosis to be the critical information around which all treatment planning was conducted while others considered diagnosis to be irrelevant.

There was one code I used 95-99 percent of clients I saw [V code]....I think the reason I did that was it didn't seem too offensive; probably the least offensive of anything I read. Plus I didn't feel like a very good judge anyway. I didn't feel as though I was very well trained at diagnosing people so I picked a code that I would hope if the client saw it wouldn't be offended by it.

Some workers use DSM-III quite effectively. I don't use it effectively at all...It seemed to be that the main impression was that people were showing up here with feelings of despondency; kind of depression kind 
of symptoms. So I thought as I went through the DSM-III, or IV now, to see what would be the least damaging diagnostic impression. I use mostly 311.00 which is depression not otherwise specified or something like that. I have a real dislike for labelling people anything so I don't like this very much.

Interestingly, even when clinicians identified that they were uncomfortable with diagnosis, they also reported that the codes submitted to MHMIS accurately reflect how they see the client.

Social workers are often considered to be adverse to labelling and nurses are often seen as medically oriented. In contrast to this stereotype, interviews were conducted with social workers who were comfortable with diagnosis and nurses who were opposed to diagnosis. Clinicians who had taken additional training in diagnosis, such as the Child and Adolescent Mental Health Worker program, were much more comfortable with diagnosis and the relationship of diagnosis to treatment.

Concern was expressed about potential liability as a result of making diagnoses.

Generally I stick to $\mathrm{V}$ codes unless the doctor has made a diagnosis then I'll put his down or hers down. A couple of times I've put my own down that was not a V code, but if I don't know I use the 799 code [not yet 
diagnosed]... One of the things you are told is whatever you put there, if you ever go to court you have to be able to back it up. If a lawyer says to you "What makes you an expert?" well you say "Nothing". So I think in that respect most people stick to the V codes because of that, they're safer.

$\mathrm{V}$ code diagnoses are found in DSM-III-R and ICD-9-CM. These diagnoses are not specific mental disorders but refer to a wide variety conditions such as relational problems, problems related to abuse or neglect, noncompliance with treatment, and school or work problems. These conditions can be the focus of treatment or they can be factors complicating treatment.

The Mental Health Division provides direction regarding the types of services which will and will not be provided. In the past, direction was given that $\mathrm{V}$ code conditions are not within the mandate of Mental Health Services. More recently the scope of Mental Health Services has been clarified and excludes marital counselling and sexual abuse counselling, although counselling in these areas is still appropriate if there is a concurrent mental health issue. For instance, a person experiencing depression as a result of marital problems would still be eligible to receive services from the program including marital therapy. Most clinicians identified that the mandate does not influence diagnosis since these clients are usually experiencing concurrent mental health problems. 
However, a number of clinicians identified that diagnosis is influenced by the mandate.

Almost any sexual abuse victim who is struggling with those issues could fit at least one of the more general diagnosis if not one of the more specific ones at a given time. If that's the case and they come in clinically depressed or classifiably anxious or whatever then we can do it [provide treatment] but from a treatment perspective it doesn't seem very proactive to do it as narrowly as we've been told we need to do it.

It [accuracy of diagnosis] is influenced to a large degree by what our mandate is. We used to be able to put posttraumatic stress disorder on this form [MHMIS Open/Close form]. One of the things I do is I work with a lot of adults who have been traumatized as kids, so I used to use that a lot. Then we were advised that we weren't to do marital counselling or sexual abuse counselling, so now they're depressed.

The presenting symptomatology, in terms of a lot of this stuff [past history of sexual abuse], is always depressive, despondent symptoms...I would see a person who presented with depressive symptomatology. Somewhere down the road, like three, four, five, six sessions down the road the person might disclose sexual abuse. At that point, if the system allowed 
it, that is where you would make your change...So you leave your diagnosis as depression not otherwise specified... You are not going to tell someone to go away after six sessions when they disclose.

Two clinicians also identified that the diagnosis they provide is based on the condition for which they are providing service. Other conditions that the client is experiencing may not be identified. For instance, the clinician may not code bipolar disorder as the diagnosis even when the person suffers from bipolar disorder if marital or family problems are the issues being addressed.

If its a Generalized Anxiety disorder then that's fine but what is the work we have to do? We are not the ones prescribing the anxiolytics or doing the medical therapies, we are doing the talk therapies. So it becomes much more important to identify the $\mathrm{V}$ code issues going on or the psychosocial things going on so in that way there is a diagnosis on file and it needs to be accurate, but I find the focus of what happens in these rooms is regardless of that in a way. We are dealing with peoples life circumstances more than what medications and other therapies that we don't do here.

Clinical records staff identified that it can take some time for diagnostic information to appear on MHMIS even if the case is already closed. Before information can be entered into MHMIS, the discharge summary has to be 
prepared, the diagnostic conference has to be done, the notes have to be typed and the chart has to be prepared for clinical records. "A patient can have a couple of admissions before I get the files to code them." Submission of diagnosis to MHMIS is usually a month behind but can be as much as four months behind.

Most clinicians and clinical records staff have now been trained in use of DSM-IV (Diagnostic and Statistical Manual of Mental Disorders, Fourth edition) and consider it an improvement in diagnostic classification. However, MHMIS does not yet accept DSM-IV diagnoses. Some clinicians identified that they invest less effort in being comprehensive with short term cases. For instance, precision of diagnosis and comprehensive demographic information may not be a priority for a client who is seen as a single contact.

\section{Contact Information}

Clinicians initial response to questions about the accuracy of contact information was often that the data was inaccurate and/or incomplete. Further investigation found that the major concerns clinicians identified may not impact on data quality.

The primary concern was that contact data did not capture the range of activities undertaken by a CMHW. For instance, time spent in preparing reports or offering a presentation at a local school was not captured. A great deal of 
work could go into nonclient activities and this information was "lost". MHMIS would capture contacts with clients but would not reflect clinician activity.

As far as client contact goes it is very accurate. How many clients you see each week and whether they are direct [client contact] or phone call, blah blah blah, all that kind of stuff is accurate but it misses a large part of our actual workload.

While information on all clinician activities may be of interest to some supervisory staff, MHMIS was designed to capture information on client services rather than clinician activity. Inability to capture clinician activity does not suggest that MHMIS data is inaccurate in capturing client services.

Concern about how MHMIS information is used is related to concerns about the ability of MHMIS to record clinician activities. Two clinicians expressed fear that MHMIS data will be used to evaluate clinicians. Information is submitted to ensure MHMIS data will not reflect poorly on the clinician at some later point. "That is the paranoia. What is the point of this. If it is to measure client progress that is one thing, if it is to measure worker efficiency or effectiveness that is something else".

The second concern was with the ability to correctly classify contacts. Some activities do not fit into a single category. For instance it is not always clear whether a contact was for collateral information or a consult since both may 
occur at the same time. In the event that there is no specific focus clinicians may enter general codes such as contact type 62 (contact service, no client contact) and therapy 901 (no therapy provided). Clinicians identified that they try to be as accurate as possible but do not invest a lot of time in trying to decide which of the codes to submit. Clinicians also identified that most codes were quite clear.

One of the factors which influences the accuracy of contact information is inconsistency of data collection between clinicians and even for the same clinician at different times. The MHMIS manual defines an contact as a "significant therapeutic or clinical intervention/assessment done on a client by a professional". However, the decision about what constitutes a significant contact varies greatly between clinicians.

Do we log everything or do we just log significant encounters [contacts]. Then you get into this what is a significant encounter. You hear some people around the regions might be every little breath they log [record every possible contact] and maybe you're doing only significant [record significant contacts] so if you're going to workload measurement what does that mean for you. I tried to look at more significant kinds of things. Where the client phoned in and said they weren't showing up for an appointment I would log them under a 'no show' but I wouldn't also 
$\log$ them as a client call where some people might do that. It [recording] can inflate or deflate a system.

Differences in what information is submitted may depend on what instruction the individual receives on orientation as well as their views on paperwork in general. Some of the charts which were reviewed contained almost no information while others contained detailed documentation such as six notes on the same day each reporting a phone call to the client and no answer.

One clinician identified that some clinicians do not submit contact information. It was also identified that information for the same clinician may be "quite sketchy if you don't have time and more complete at other times". There is workers that just refuse to do them [contact forms]; they just don't fill them out. So as far as the system is concerned they haven't seen anybody in five years. Or lets say you fill them out up until April and then for some reason you're ill in May or you have an erratic caseload where there's no possible way you can fill them [contact information] out and then you're so far behind...you just let those go to the wayside for a couple of weeks. So then it looks like you've never seen your clients in the whole month of May.

Clinicians consistently reported that virtually all direct client contacts were captured. Some phone calls and some informal consultations would be missed. 
The number of calls and consults that are missed often depends on the amount of time between the event and recording of the event. Several clinicians identified that they keep a running list of events that occur during the day. This list may be kept on a piece of paper on the desk or in an appointment book. This information is then transferred to MHMIS and client charts at the end of the day or week. When recording is done as events occur, only a small number of contacts are missed. Other clinicians do not record events as they occur and/or submit MHMIS information when they have time. One clinician identified being about five months behind on contact sheets. When time is available MHMIS contact information are submitted based on information in the appointment book. This clinician estimates that 25 percent of telephone calls are missed but believes that virtually all direct client contact is captured.

Clinicians differed greatly in their views about where information on client contacts should be captured and the frequency with which recording should be done. Some clinicians identified that they consistently write notes on the client chart identifying the date the client was seen and what was done. More often, and particularly in one region, clinicians expressed the view that recording all information in the client chart would take too long and would not be beneficial to the clients. Chart notes tend to summarize contacts over a period of time. In fact, several clinicians identified that MHMIS contains more accurate information 
on client contacts than client charts.

I don't always document on the file, but I kind of count on the encounter [contact] sheets to document what's been done between [initial assessment and termination summary]. I think we're supposed to write them [chart notes] every so often, every three months at the least but I tend to not do that and use the encounter [contact information] to portray what has been happening.

So in terms of who I see, in terms of clients during the day or during the week, it [MHMIS] is accurate in terms of the number of people I see. However it doesn't translate into the files. For example if $\mathbb{I}$ saw somebody three or four times a week, at the end of the month or whenever I decide to do the recording I probably just did one recording. A progress note as to what happened over that two or three weeks. So you wouldn't see it recorded every day [in the chart] as it is here [MHMIS contact form]. So this [MHMIS contact form] is the most accurate thing I have.

The MHMIS form is even used by some clinicians as a means to enhance chart notations. "It has also become a very useful way for me to keep track of the people I've seen and being able to remember what I've seen them about." 
Another influence on completeness of MHMIS contact information is workload. Several clinicians expressed the view that workload influences the amount of information submitted. However these same clinicians were unanimous in suggesting that all significant contacts related to the client are captured in MHMIS.

Caseloads are very high and there is a lot of paperwork and I think people get bogged down. I think you really have to have good time management skills at any time but when you're working in a fairly independent functioning capacity where there is not someone checking up on you on a daily basis... Some people really get behind and then you get to the point where like why bother any more, they're not accurate. You have to somehow discipline yourself. But that's easy to say. Then you've got to priorize what are your priorities for the day. Are they meeting the clients needs or the paperwork needs.

Clerical staff in one facility identify that they submit contact information for the medical staff based on the dictation provided by the medical staff. If the therapy provided is not clear, the clerical staff seeks clarification prior to entry. In this instance there should be a direct correspondence between chart notations and MHMIS contacts. 


\section{Therapy Information}

MHMIS contains therapy information on the Open/Close form. When a case is opened at least one therapy, such as diagnostic assessment or psychodynamic assessment, is identified. If the clinician knows what therapies will be provided, or the general areas they are going to be helping the client with, these codes may also be entered. Additional therapies can be added when the case is closed. Although it is possible to update the therapy codes during an episode of care through a clinical status change is rarely done. Therapy information is also submitted with contact information. Up to three therapy codes can also be provided for each client contact.

One clinician identified that therapy information on the Open/Close form would be quite similar to therapy information on the contact forms but most clinicians felt that the therapy information in the contact data was most accurate. However, therapy information provided with contact information can be no more complete than the contact information. For instance, therapy information associated with an contact that is not entered is lost.

Generally, clinicians felt that the therapy information submitted reflected the services that were provided. One clinician identified that the therapy codes are fairly broad and that a range of codes was used to describe therapies provided to clients. However, several clinicians identified that they regularly use specific 
therapy codes that described their approach rather than trying to analyze the process of each contact. For instance, a clinician who works with children might primarily use play therapy and systems approach for work with families.

It's hard to understand what the value is in being too inclusive or too exclusive or too broad with these things. I can agree with all these things and a mixture of just about everything is done just about all the time so I pick a few favourites that seem to work well for me.

Conversely, another clinician felt that there were insufficient therapies codes to describe the work that is done and that, as a result, the other category is used a great deal.

Although definitions of each therapy are provided in the MHMIS manual and although many clinicians have reviewed the definitions, it was felt that assignment of therapy codes was subjective.

Interpretations I think probably vary amongst workers. What I would put down if I was to have a session with a client and code it whether it was seen as a treatment session or a supportive session, I could see maybe that might be pretty much to the individual how they interpret how they saw that particular session.

\section{Open Date and Close Date}

According to the MHMIS manual, the open date is the date when formal 
service is initiated. For inpatient cases the open date is the date of admission and is easily identified. In contrast, outpatients and regional services cases may be opened as of the date the referral is received, the date the client is first seen, or after the assessment is completed. Cases may also be opened on the basis of substantial collateral information or a telephone contact with significant interventions. Cases are usually opened as of the date the client is first seen.

Some region/facilities have an intake worker who conducts an initial assessment prior to assigning the client to a clinician. A client who is seen by the intake worker may be opened to the intake worker and then transferred trough a clinical status change or the case may be opened by the clinician once the case has been assigned.

A small amount of work may be done with clients prior to the time a client is first seen. Collateral information may be obtained and, according to one clinician, the case may be screened to determine whether the client is interested in receiving service and will be "compliant". This clinician suggested there was too much paperwork to open a case if the client was going to refuse service.

The information required to open a case may not be submitted for entry to MHMIS for a period of time after the case is active. MHMIS still accepts the information, including the open date as identified by the clinician, but contact information can not be entered until after the case is entered into the MHMIS 
system; Contact information must be held until it can be entered. As a result there is substantial pressure to open the case rapidly. Clerical staff at one outpatient facility reported opening a case rapidly was also important so that clinicians are not duplicating efforts of another clinician, for instance a regional clinician already working with the client. In inpatient services a new case is entered into MHMIS within a few days although changes to diagnosis may be made at a later time. Regional and outpatient cases are entered into MHMIS later than inpatient cases since clinicians may be quite dispersed in the region and may have to wait for sufficient information to open the case.

Inappropriate cases are not opened and are referred elsewhere. For instance a client who is best served by another program such as Child and Family Services or vocational rehabilitation programs or an individual whose issues are primarily legal, such as a pending court case, may not be opened. Intake workers may spend a substantial amount of time conducting assessments of cases that are not opened. One intake worker identified that 25 percent of referred cases are not opened, partially because the referrals were "doctored". Referring organizations know what the program mandate is and tailor the referral to fit the mandate but the client may bear little resemblance to the referral.

Clinicians consistently reported that any client viewed as a mental health case is opened in the MHMIS system. Even cases which will not be followed, 
such as a single contact or a consultation, are opened since they are often referred again later on. One clinician identified that individuals who legitimately require service but are peripheral to the mandate may not be served if caseloads are high.

According to the MHMIS manual, the close date is the date on which formal mental health services are terminated. As with the open date, close date for inpatients is the date of discharge and is easily identified. For regional and outpatient clients the closure date is less clear. In some cases closure is mutually decided between the clinician and the client. When this occurs the case is usually closed as of the last date of contact or the date charting is completed. More often, however, clients fall out of service and the case may become inactive after a period of time. For instance the client may miss appointments and not reschedule. The date of closure for cases which have become inactive is less clear.

Most clinicians identified that cases which become inactive were closed within three to six months. Some region/facilities have feedback mechanisms which facilitate timely closure of cases. The feedback mechanisms which are used include review of inactive cases and direct supervision by managers. The review process involves bringing cases which have not been seen in the previous three to six months to the clinicians attention for review. At that point the clinician can either close the case, contact the client to see if they wish to 
terminate or the clinician may decide to keep the case open. Supervision involves discussion of case lists with the clinician to determine activity with each client and discuss closure of cases which are inactive. Some clinicians also receive case lists periodically and use the list to identify cases which have become inactive. In contrast, some region/facilities have no process to prompt closure of cases, although clinicians are still encouraged to close cases as soon as possible.

Several clinicians identified that they follow up on inactive clients by telephone or with a letter prior to closing the case. For instance, the client may receive a letter advising the client to contact for an appointment. If service is not required the case is closed. Contacting the client may mean that an inactive case remains open for an additional two months.

Several clinicians stated that they are reluctant to close cases. If there is a suspicion that the client will come back into service within a short period of time or that follow up may be required, it is useful to keep the case open. Reopening a case involves substantial paperwork and contacts can not be entered into MHMIS if the case is not open.

One region identified that a form was developed to "append" information to a closed case. Although the form used to append cases is different from the MHMIS Open/Close form and is simpler for the clinician to complete, MHMIS captures the information as a new case. If this form is used for a client that is 
seen once a week for a month after the case was closed, MHMIS would contain five separate episodes for the client.

One region and some programs in another region prepare a termination summary at the time a case is closed. The termination summary outlines the reason the client came into service, what interventions were implemented and what the outcomes were. Clinicians found the termination summaries useful, particularly if the client came back into service, but found it difficult to find the time to prepare the summary. This often led to a delay of up to six months in closing the case.

I have to say that my files don't always get closed at an appropriate time because I don't necessarily get to it. I probably could fill out the [MHMIS Close] form and send my termination summary later; I guess that would be more accurate. What I tend to do is do the whole package and send it off for typing when I get around to it.

Clinicians also identified that the length of time in service and intensity of service does not necessarily reflect the level of symptomatology experienced by the client. The length and intensity of service also depends on what other agencies are involved with the client and whether the client declines services.

\section{Change of Status Information}

While status changes can be submitted for almost all fields (changes to 
birth date or name must go through the central registry), there is consensus amongst those interviewed that changes are usually only made for diagnosis, change in case status from active to maintenance, or change of clinician. Clerical staff in one facility identified that they check current address with clients as they come in for appointments but clinical staff submit few address changes. Some changes to the information are simply documented when the case is closed. For instance, a change in diagnosis, addition of diagnoses or addition of therapy codes may be added at the time the case is closed rather than submitting a Change of Status form.

Almost all status changes are initiated by clinical records or clerical staff. Some clinicians stated that they were not familiar with the form and others do not use the Change of Status form although it was considered important that the information be current.

Sometimes people don't fill out the change form, and that can be a problem because if they [clients] move and the change form is not on there [MHMIS form] now you don't know where this person has moved to and now you have to go through the notes to find out where they've moved to. I think the MHMIS form is very useful when its used in conjunction with the other tools. It is not useful if its not updated. 
I find that once you've done them [diagnosis] you don't necessarily go back and change them...That's one of the things that I think maybe we need to change... When you go to use that information again...that part of it [diagnosis] is very important.

\section{Uses of MHMIS Information}

Most of the clinicians who were interviewed used MHMIS information in some fashion. A small number used computerized information to look up the PHIN for clients. For instance, the client would be asked for full name and birth date when the initial appointment was made and, using MHMIS, several pieces of information such as PHIN and the Manitoba Health Services registration number could be obtained prior to seeing the client. Information may also be obtained from MHMIS identifying whether the client has received mental health services and where those services were delivered. If the client has previously been seen in the region, information on previous episodes of care and the number of times a client had been seen could also be obtained. This information was seen as useful for continuity of care as well as for establishing a pattern of service. For instance, the information may provide a point of discussion for clients that have repeatedly dropped out of service to determine why this occurs. However, most clinicians had no access to MHMIS information and/or no knowledge about how to access it. In some region/facilities only a limited 
number of personnel have access. Even when clinicians have passwords, they may not have ready access to the equipment.

We don't have as much access to computers as I would like in terms of accessing my own material. I mean its very hard to get on the screens because there is maybe two screens in the offices and you've got all these programs. The secretary needs the machine.

A few clinicians identified that they use the MHMIS Open/Close form for reference and suggested that it was a "focal point" for information. However several clinicians, particularly those who do not code their own information, felt that the form was not useful for this purpose since the information was coded rather than in writing. For instance, marital status is more easily understood if married appears in the field than if 02 appears. "The system that we had before was more helpful in the face sheet being user-friendly. It gave almost psychosocial stuff quicker and in a more reasonable way".

Clinicians more frequently use MHMIS data in the form of client lists and lists of inactive cases. These lists are periodically provided to clinicians in some region/facilities. Clinicians usually found this information useful in identifying cases which had not been opened and to review which cases should be closed. One clinician felt the lists were prepared by management to "monitor" compliance with paperwork. 
Supervisory staff in some region/facilities appear to be using MHMIS information for purposes such as selecting cases for audit or preparing statistics. Specific questions may be asked such as the average length of stay in a facility or the number of clients readmitted within two weeks of discharge. Questions may also be asked about the number of intakes in each area of the region and the number of contacts to facilitate manpower planning. However, it appears that these analyses are conducted by entering MHMIS information on another system or by manually tallying the information rather than manipulating MHMIS information directly.

The number of individuals who are trained in retrieving information from MHMIS is very small and none of these individuals were interviewed. Four of the individuals interviewed had made attempts to retrieve information from MHMIS and expressed frustration at not being able to do so. For instance, a list of clients in a particular program must be prepared manually or a special request must be made to the Mental Health Division rather than printing off the information.

It's not doing what we need right now. We have several programs and the only way I can pull off information is by provider or by heath unit when what I need is to be able to pull it off by program.

Similarly, it is not possible to identify the number or type of contacts for each 
clinician without printing up all the contact data and manually tallying the information. Attempts to make the system more responsive have not been successful.

Clinicians in certain region/facilities expressed concern that MHMIS information is, or will be, used for workload management. Since MHMIS does not capture information on the amount of time spent at each contact and does not capture nonclient activities it is not particularly useful for workload management. One region/facility wants information for workload management and is therefore entering all information twice, once on MHMIS and once on their own database. "Attempts by administration to get some value from the stats were really shortcircuited by the design. It was not designed for that [workload measurement]". We are double entering is what we're doing. We enter the information on MHMIS and then we take the same information and we enter it on [our own program]. I think if we could get that first part from MHMIS it would cut our workload down dramatically. It would save us an enormous amount of time.

One other region is also maintaining a separate "rotary system" of information on clients. The rotary system contains cards with minimal information, such as diagnosis and case manager, on clients that have been served. The separate system is maintained because it is considered to be more 
accurate than MHMIS. "We are doing two systems at one time...The manual system they have not wanted to delete because they have felt that the MHMIS system isn't adequate at this time".

Double recording impacts on enthusiasm for the system. "When you've got two systems operating it makes it hard for people to buy into one". Ironically, the additional effort required by two systems may indeed undermine accuracy of information in MHMIS due to the volume of paperwork and the perception that the information in MHMIS is already inaccurate. For instance, one clinician identified that opening a case involves filling out both the MHMIS Open/Close form, the Stats Transmittal form and an initial assessment form. Transfer of a case to another clinician or change in diagnosis involved the Stats Transmittal form only and closing a case requires both MHMIS Close and Stats Transmittal forms. This volume of duplicate paperwork makes it easy to omit pieces. In particular, failure to submit the Change of Status form to MHMIS for transfer of a case left this clinician with inaccurate case lists and confirmed the impression that MHMIS is inaccurate. Another clinician from the same region/facility identified that if MHMIS was accurate it would be more useful since the rotary system does not provide information on when the client was last seen, the diagnosis is not as complete, therapy information is not provided and so forth. 
One clinical records staff felt that there was unnecessary duplication of information in client charts.

We could use the MHMIS system for what we are using our system down here for and I understood that that was the intent when it was first implemented,...this would be the end of the admission papers. We would just have one form to complete, like the statistics...that was the idea in the first place.

Most clinicians view MHMIS as primarily administrative and have not considered how the information could be used for client service. Two clinicians reported that the codes were medically and facility oriented rather than being community oriented. For instance, the codes available to describe closure of a case include several types of discharge, death, transfer to another facility or moving to another region or province. These codes are not useful to describe case closure in a community setting.

\section{Other Issues}

The MHMIS Open/Close form contains three fields for medical diagnoses and five fields for therapies. On some units at one facility the number of medical diagnoses and the number of therapies identified drastically exceeds this number. Clinical records staff do not have sufficient information to prioritize this information and therefore attempt to enter all the information into MHMIS using 
a series of status changes. For instance, if there were 15 therapies specified, a status change entering five new therapies would be submitted to MHMIS on each of the three days prior to discharge. The clinical records staff in this facility also identified that there may be more clinicians involved in a case than can be captured in existing fields. For instance, in addition to the case manager, a client may have a psychological assessment, dietary assessment, social history and other professional involvement. The decision about which clinicians would be entered as primary and secondary therapists is arbitrary. Clinical records staff expressed a desire for more fields for entry of this information.

One clinician expressed concern about the investment of time and effort in MHMIS when the information serves no apparent purpose.

There has been a sense that there is very little either loyalty or investment in the system by the people who know and people who care. To invest more in something when there is very poor return already is not the best use of my time certainly or our time to agitate [to improve MHMIS].

This concern was also expressed in preliminary interviews. The individual who was interviewed reported that the area had not submitted contact information in over a year and concluded "no one had noticed or cared. It can't be that important".

Some clinicians identified that they were "compulsive" about paperwork 
while others "generate as little paperwork as possible". The attitude toward documentation likely affects both MHMIS and client charts and, as a result, is not likely to affect the reliability of MHMIS data. However, these differences may affect validity of contact data and completeness of optional MHMIS information.

The attitude of managers toward documentation appears to have a very strong effect on the perspective of clinicians. Managers who consider documentation to be important communicate this view to staff and the view is largely adopted. Even though some individual differences remain, there appears to be pressure to comply. In particular, if a supervisor is using the information for supervision of caseloads, clinicians ensure the information is as accurate as possible.

Clinicians share information and a degree of consistency develops between clinicians in the same area. For instance, individuals in one region consistently held the view that recording of all contacts in client charts was not beneficial to clients and was not an effective use of their time. While there is some consistency within an area, the method of collecting and documenting client information can differ greatly between region/facilities. For instance, one region has a standard assessment form, a second region uses specific format for assessment notes and the third region allows individual clinicians to select the assessment method and documentation format. Protocols for review of cases also 
differs. Some region/facilities have formal reviews at least once per year where the client is assessed and diagnosis and treatment are reviewed. Since information that is collected as part of the assessment is easily available, it is more likely to be submitted to MHMIS. Regular review of cases increases the likelihood that updated information will be submitted to MHMIS. In addition, feedback mechanisms such as prompts for review of inactive cases, routine provision of client lists to clinicians and supervision of cases all support data accuracy.

Certain clinicians expressed concerns that diagnoses were not as precise as they could be. Despite this concern, it is probable that their diagnoses are more precise than other diagnoses made in the community. CMHW submit information on diagnosis after a lengthy assessment where other practitioners, such as primary care physicians, have a considerably shorter period of time to make this determination.

\section{Chart Reviews}

A total of 140 client charts were reviewed including 60 charts from three regional services, 40 charts from two inpatient services and 40 charts from two outpatient services. Two charts could not be located in the region/facility and one chart was not reviewed because the client was known to the researcher. In total, three client charts were not available for review. When a chart was not available, 
the next chart on the list was selected for review.

\section{Demographic Data}

The accuracy of demographic information was determined by comparing chart information to MHMIS data as of the date the case was closed or as of the date of extraction if the case was open at the end of the study period. It is possible that changes in demographic information occurred between the end of the study period and the date that the information was extracted by Manitoba Health. However, demographic information can not be obtained as of a specific date. It is anticipated the discrepancy in dates had minimal impact on agreement since changes in demographic data are infrequent.

Demographic information includes both required and optional information on each case. Information is collected from the client and entered on the MHMIS Open/Close form when a case is opened. Required information includes Manitoba Health Services registration number, PHIN, name, gender, birth date, address and marital status. Optional information includes Social Insurance Number, Department of Veterans Affairs number, treaty/innuit number, social allowance number, birth place, religion, highest level of education achieved, employment status (employed, unemployed, student, retired etcetera) and occupation. Changes in demographic information can be submitted at any time when the case is open and an optional field is available for forwarding address at 
close.

The required demographic fields are linked to Manitoba Health registry data. This means that complete demographic information as contained in the registry can be retrieved with only partial information such as surname and birth date. If the client presents with information that differs from registry data, the registration information can be changed by supplying information and supporting documentation, such as a marriage licence or divorce decree, to Manitoba Health. Since changing the registration information can take some time, chart information can temporarily differ from the registry and hence from MHMIS data.

\section{Detailed Methods for Demographic Information}

For confidentiality reasons, some of the information contained in MHMIS is not in the Manitoba Health Research Database. Data fields containing personal identifiers such as name, address and social insurance number are not present on the Research Database. Manitoba Health Service registration number and PHIN numbers are present but are in scrambled form to ensure that individuals cannot be identified. Since analysis of MHMIS data is conducted using data in the Research Database, analysis was limited to information available on the Research Data Base. The specific variables which were analyzed include gender, date of birth, marital status and postal code which are required fields. Optional information on education, employment, occupation, treaty status and religion were 
also analyzed.

The frequency with which demographic data was obtained from the MHMIS Open/Close form differs between demographic fields (see Table 1). Information on gender was available from other information on the client chart in every case while twenty percent of the information on education was available only from the MHMIS Open/Close form. At least one demographic variable was obtained from the MHMIS Open/Close form in 71 out of 140 reviewed charts.

Some of the information in MHMIS is more detailed than client chart information. For instance, it was often difficult to determine on the basis of chart information the number of years of postsecondary education a client had achieved. The chart would refer to a certificate from a community college or completion of a program of study while MHMIS contains information on the number of years of postsecondary education. For this analysis, comparison was limited to whether or not the client had had postsecondary education rather than analysing the number of years of postsecondary education.

Insert Table 1 about here

Similarly, employment is captured in MHMIS using the following categories; never employed, retired, student, employed full-time, employed part-time, 
Table 1

Source of demographic information abstracted from client charts

\begin{tabular}{lcc}
\hline Demographic variable & $\begin{array}{c}\text { MHMIS Open/Close } \\
\text { form }\end{array}$ & Other site in client chart \\
& N (\%) & N (\%) \\
\hline Gender & $0(0.0)$ & $140(100.0)$ \\
Date of birth & $5(3.6)$ & $135(96.4)$ \\
Marital status & $9(6.4)$ & $131(93.6)$ \\
Postal code & $22(15.7)$ & $118(84.3)$ \\
Education & $28(20.0)$ & $112(80.0)$ \\
Employment & $31(22.1)$ & $109(77.9)$ \\
Occupation & $24(17.1)$ & $116(82.9)$ \\
Treaty status & $0(0.0)$ & $140(100.0)$ \\
Religion & $19(13.6)$ & $121(86.4)$ \\
\hline
\end{tabular}


sheltered employment, unemployed, self-employed and not applicable. This level of specificity is not available on most client charts. For this analysis, the categories were collapsed to form five categories namely employed, retired, student, unemployed and other employment which included sheltered employment and training.

General Linear Modelling was used to determine whether agreement on required demographic variables was related to clinician type, service type or region/facility. The aggregate or single summary measure for demographic variables was prepared using the proportion of agreement on required demographic information, namely gender, date of birth, marital status and postal code. For example, a client whose MHMIS and chart information matched on three out of four variables was assigned an aggregate value of 0.75 while and client whose information matched on two of the four variables was assigned a value of 0.5 . Nodifference in agreement was found $(\mathrm{F}=1.53$ with $16,123 \mathrm{df}, \mathrm{p}<0.10)$ related to clinician type, service type or region/facility.

Separate investigations of each demographic variable were undertaken to determine the extent to which information was present in each data source and the extent to which the data sources agreed.

\section{$\underline{\text { Results for Demographic Information }}$}

Required information. On all 140 cases reviewed, gender according to client 
charts was the same as gender according to MHMIS (see Table 2). Date of birth according to MHMIS and according to client charts is in agreement 83.6 percent of the time. Although date of birth is a required field in MHMIS, it is not always complete. Four cases (2.9\%) were complete on the chart while only partial information, such as year and month, was available on MHMIS. Three cases (2.1\%) were found on MHMIS and not on the client charts. A total of 16 cases (11.4\%) disagreed on date of birth. Most disagreements on date of birth are minor and represent a difference of days (eight out of 16 cases). In every case, the date of birth on client charts was within three years of registry age. Most discrepancies arise from a single digit difference in year, month or date or from transposing month and date.

Insert Table 2 about here

Marital status has nine possible categories including never married, married, common-law, separated, divorced, widowed, other and unknown. These categories are not mutually exclusive. For instance, one case involved an individual who was divorced and living common-law with another person. In this instance, a MHMIS code for either of these possibilities was considered a match. MHMIS and client charts agreed on marital status in 119 cases (86.2\%) including two cases which were missing the information on both files. 
Table 2

\section{Agreement between MHMIS and client charts on demographic variables}

\begin{tabular}{|c|c|c|c|c|c|c|}
\hline \multirow{4}{*}{$\begin{array}{l}\text { Demographic } \\
\text { variable }\end{array}$} & \multirow{4}{*}{$\begin{array}{l}\text { Both } \\
\text { missing }\end{array}$} & \multicolumn{3}{|c|}{ Disagree } & \multirow{4}{*}{$\begin{array}{c}\text { Agree } \\
\text { Chart \& } \\
\text { MHMIS } \\
\text { match }\end{array}$} & \multirow{4}{*}{$\begin{array}{c}\text { Percent } \\
\text { agreement }\end{array}$} \\
\hline & & MHMIS & Chart & Chart \& & & \\
\hline & & only & only & MHMIS & & \\
\hline & & & & disagree & & \\
\hline Gender & 0 & 0 & 0 & 0 & 140 & 100.0 \\
\hline Date of birth & 0 & 3 & 4 & 16 & 117 & 83.6 \\
\hline Marital status & 2 & 3 & 3 & 13 & 119 & 86.2 \\
\hline Postal code & 2 & 28 & 1 & 28 & 81 & 58.7 \\
\hline Education & 31 & 3 & 22 & 12 & 72 & 66.1 \\
\hline Post sec ed ${ }^{\mathrm{h}}$ & 129 & 0 & 6 & 0 & 5 & 45.5 \\
\hline Employment & 11 & 5 & 35 & 13 & 76 & 58.9 \\
\hline Occupation & 54 & 18 & 23 & 5 & 40 & 46.5 \\
\hline Treaty status & 130 & 3 & 2 & 0 & 5 & 50.0 \\
\hline Religion & 72 & 3 & 11 & 4 & 50 & 73.5 \\
\hline
\end{tabular}

a Information missing on both data sets has been excluded from calculation of agreement

h Post secondary education 
Since address is not contained on the Manitoba Health Research Data Base, postal code is used to provide an indication of location of residence. MHMIS postal code was a complete match in 81 cases $(58.7 \%)$. Of the 57 cases that did not match, almost one half (28) were missing postal code on the client chart.

Optional information. Education is divided into two variables in MHMIS, namely, highest level of education achieved (kindergarten to grade 13) and number of years of postsecondary education. Information on highest levelof education achieved was present on at least one file in 109 cases $(77.9 \%)$. Of the cases which contained education information, 66.1 percent agreed on the grade level. More information was found in client charts than was found in MHMIS. Disagreement between MHMIS and client charts occurred in 12 cases and were usually limited to discrepancies of one or two years. Charts suggest that eleven individuals have some postsecondary education. Only five of these individuals had corresponding information in MHMIS.

\section{Issues Regarding Demographic Information}

Required demographic information on date of birth, gender and maritai status contained in MHMIS matches quite closely with information contained on client charts. Since this information is linked to the registry, MHMIS may be more accurate than client charts for these fields. However, the registry does not 
always contain the most recent information on client address.

Some demographic variables are much better captured on one data source than another. For instance, postal code was present only in MHMIS on 28 occasions. Alternately, employment and education information is more frequently found in charts. It is not possible to determine from this data whether the information found only in one data source is accurate. However, it is probable that the accuracy of information contained in MHMIS is higher than demonstrated by a match between data sources. Postal code information was submitted to MHMIS much more frequently than it was recorded in client charts.

Optional information was either missing or found only on client charts about sixty percent of the time. Even with good agreement between data sources, a variable is of little value for analysis if information is incomplete. If information on education, employment, religion and so forth is considered valuable, it may need to become a required variable. Otherwise, there may be little benefit to collecting the information.

Other factors which may influence accuracy of postal code information include admission to a facility from a temporary residence and inaccurate postal codes presented to clinician at interview. It was observed during the chart reviews that a large number of clients admitted to inpatient services at mental health facilities were admitted from a temporary residence such as a family 
member's home. A temporary living arrangement would not likely be reported to Manitoba Health and may never be captured in registry data. Registry information such as birthdate and postal code overrides information submitted by the client and can not be changed by regional or facility staff. Thus, the correct address may not be reflected in information captured in MHMIS.

It was also observed that many of the discrepancies in postal code between MHMIS and client charts were the result of a single digit difference. It is possible that inaccurate postal codes are provided by the client or that the codes are documented incorrectly.

\section{$\underline{\text { Diagnosis }}$}

The source of diagnosis and process for coding and entering the data varies a great deal depending on the whether the client is served as an inpatient, outpatient or by regional services. In regional services, the diagnostic codes are selected by the CMHW. The diagnosis is made independently unless a referral is received from a physician or a facility. If a referral contains an established diagnosis made by a physician, the referral diagnosis is entered on the chart and in MHMIS. In addition, the MHMIS field indicating that the diagnosis was made by a physician is marked. More often, the CMHW enters their diagnostic impression in the client chart, and codes and enters the information on the MHMIS Open/Close form for entry by clerical staff. 
Diagnosis is recorded on the client chart at several different times for inpatients at a mental health facility. Diagnostic impression at admission is recorded on the admission forms, diagnosis is established at a diagnostic conference held shortly after admission and diagnosis is again established at a discharge conference. Diagnosis may also be reviewed and either changed or confirmed at periodic case reviews. The diagnostic impression at admission is determined by the admitting physician. Conference diagnoses are established by a multidisciplinary team headed by a physician or psychiatrist. In mental health facilities, information for the MHMIS Open/Close form is abstracted, coded and entered by clinical records staff. The process of determining a diagnosis and having it entered into MHMIS for outpatients is similar to regional services, although many more clients come directly from the mental health facility with an established diagnosis.

Diagnosis can be made and entered using either DSM-III-R or ICD-9-CM. If a DSM-III-R diagnosis is entered, the MHMIS software converts the codes into ICD-9-CM codes so that the fields containing ICD-9-CM codes are complete. In the event that a DSM-III-R code can not be directly translated (for instance if a single DSM-III-R code encompasses more than one ICD-9-CM code), it is returned to the clinician for clarification. In the regions and facilities studied, DSM-III-R was the primary diagnostic instrument. Only one facility coded ICD- 
9-CM directly; in the other facility and in the regions, DSM-III-R diagnoses were coded and entered.

MHMIS has a total of six fields for psychiatric diagnosis and three fields for medical diagnosis. Medical diagnoses are not examined in this study. The fields for psychiatric diagnosis are designated as either primary or secondary, with three fields available for each. One primary diagnosis is required by MHMIS. The other five fields are often empty, particularly for regional services cases. If the diagnosis is originally made using DSM-III-R the primary diagnoses are Axis I diagnoses (clinical disorder that is the focus of treatment) and secondary diagnoses are Axis II diagnoses (personality disorders). However, this method of organization may not transfer into MHMIS. If a client has only an Axis II diagnosis or a $\mathrm{V}$ code diagnosis, the diagnosis would appear in MHMIS as the required primary diagnosis. In short, the primary diagnosis is the most serious diagnosis and there are no distinguishing features between subsequent primary diagnoses and secondary diagnoses which can be considered comorbid diagnoses.

Status change forms are submitted when there is a change of diagnosis during the time period a case is open. For instance, if a case review finds that the existing diagnosis is inaccurate, a change of status form is completed and the new information is entered. For the 140 client charts reviewed, a total of 36 
status changes on 21 individuals were submitted to MHMIS. The majority of the status changes were for inpatients at mental health facilities.

\section{Detailed Methods for Diagnosis}

Accuracy of diagnosis was determined by comparing current chart diagnosis with MHMIS information as the date the case was closed or as of April 30,1995 , if the case was still open at the end of the study period. Agreement on diagnosis was assessed using ICD-9-CM since this field is complete whereas DSM-III-R diagnosis is often missing.

Chart diagnosis was abstracted from client charts. Diagnosis from the discharge conference was used for clients in mental health facilities when the case was closed. Cases which were open were examined for the most recent conference at which diagnosis was reviewed. For regional clients, records for the current episode were reviewed and the diagnosis at the most recent assessment was recorded.

Coding chart diagnoses was often facilitated by the clinicians. In many cases the code number was recorded in the client chart as well as the written diagnosis. Each recorded diagnostic code was compared to the ICD-9-CM diagnosis for the code to ensure that it accurately reflected the chart diagnosis. If chart diagnoses were made using DSM-III-R, an additional check was completed to ensure that coding changes in conversion to ICD-9-CM were 
captured. In cases where the code numbers had not been documented in the chart, MHMIS diagnosis was compared to chart diagnosis. If MHMIS diagnostic code matched chart diagnosis then the code number was entered as the chart diagnosis. In the event that the MHMIS code did not match the chart diagnosis, the chart diagnostic codes were selected from the ICD-9-CM manual. For instance, if the client chart stated the client suffered from moderate mental retardation (ICD-9-CM 318) and the MHMIS diagnosis code reflected mild mental retardation (ICD-9-CM 317), the chart was coded as 318. Similarly, if a chart showed bipolar disorder currently manic and MHMIS was coded as bipolar disorder currently depressed, the codes entered for the client chart would reflect mania.

As noted above, there are no conventions for the placement of diagnoses in MHMIS forms other than the first primary diagnosis. Comorbid diagnoses can be placed in more than one field. Each chart diagnosis beyond the first primary diagnosis was placed in the same field as MHMIS if the same diagnosis was present in the client chart and in MHMIS. In the event that chart diagnoses were not found in MHMIS, the diagnosis could be placed either opposite a MHMIS diagnosis or opposite an empty field. The diagnosis was placed in an empty field if the chart information was quite different from MHMIS but in the same field if the diagnoses clearly conflicted. For instance, a single client can not have both 
mild and moderate mental retardation. These codes would be placed opposite one another and would appear as a conflict. However, a V code in one file and a personality disorder in the other would be placed in separate fields since each file contains unique information. While decisions about placement of chart diagnoses affects how disagreement is described, it does not influence agreement between data sources.

Diagnostic information on six client charts was found only on the MHMIS Open/Close form. Therefore, for these six cases, diagnosis from the Open/Close form was recorded as the chart diagnosis. Two charts contained no diagnostic information. The diagnoses for these charts were recorded as missing.

MHMIS contains information on client diagnosis and changes in diagnosis over time. The ability to update the diagnosis assigned when the case was opened allows examination of client diagnosis at any point in time. Information from status changes occurring during the episode and during the study period were used to update information on diagnosis. Diagnoses in the client chart and in MHMIS were compared as of the close date for cases that were closed. Records for cases that were still open on April 30, 1995 were compared as of this date.

Comparisons were made between the MHMIS and chart diagnoses at the five digit and three digit levels. Comparisons using mental health categories were also made. Broad categories of disorder are sometimes used as a means to 
improve agreement on diagnoses. The categories used here are similar to those used by Tataryn et al. (1994) but use a separate category for substance abuse disorders and include a category for cases with no classifiable disorder. The primary diagnosis was used to place each case in one of five categories: psychotic, nonpsychotic, substance abuse, other mental disorder and no classifiable disorder. The psychotic category (ICD-9-CM 295-299) includes schizophrenic disorders, paranoid conditions and major depressions. The category of nonpsychotic disorder (ICD-9-CM 300-301, 306-309, 311) combines mild affective disturbance and neurotic and personality disorders (Tataryn et al., 1994). Individuals classified as having a substance abuse disorder (ICD-9-CM 291-292, 303-305) had diagnoses for drug or alcoholic psychosis, dependence or abuse of drugs. The 'other' mental disorders (ICD-9-CM 290, 293-294, 302, $310,317-319)$ combines a heterogenous group of conditions including paediatric disorders not elsewhere classified, organic states, sexual deviations and mental retardation. The final category includes individuals who have not yet been diagnosed or have a $\mathrm{V}$ code diagnosis. In addition, all diagnoses were hierarchically categorized into the above five categories to determine whether there are benefits to using all diagnostic information rather than primary diagnosis alone. 


\section{$\underline{\text { Results for Diagnosis }}$}

Primary diagnosis. The primary diagnosis found on client charts matched MHMIS diagnosis 76.4 percent of the time at the five digit level (Table 3) and 82.9 percent if a three digit diagnosis was used (Table 4). For instance, mania rather than depression may be coded for an individual who suffers from bipolar disorder. This type of error does not pose a problem unless precision beyond the three digit level is required.

Insert Table 3 and 4 about here

Disagreement at the three digit level resulted from entry error, diagnostic conflict and failure to update MHMIS (Table 5). In one case the discharge abstract and MHMIS Open/Close form reflect ICD-9-CM 2989 but MHMIS shows 2939 suggesting entry error.

Insert Table 5 about here

Three cases showed conflict between MHMIS and chart diagnosis. The remaining cases of disagreement $(18 / 22)$ resulted from failure to update MHMIS when a client diagnosis changes. The impact of this failure depends on the nature 
Table 3

Agreement between MHMIS and client charts on ICD-9-CM psychiatric diagnosis at the five digit level

\begin{tabular}{|c|c|c|c|c|c|}
\hline \multirow[b]{2}{*}{ Diagnostic field } & \multirow[b]{2}{*}{$\begin{array}{c}\text { Both } \\
\text { missing }\end{array}$} & \multicolumn{3}{|c|}{ Disagree } & \multirow{2}{*}{$\begin{array}{c}\text { Agree } \\
\text { Chart \& } \\
\text { MHMIS } \\
\text { match }\end{array}$} \\
\hline & & $\begin{array}{c}\text { MHMIS } \\
\text { only }\end{array}$ & $\begin{array}{l}\text { Chart } \\
\text { only }\end{array}$ & $\begin{array}{l}\text { Chart \& } \\
\text { MHMIS } \\
\text { disagree }\end{array}$ & \\
\hline \multicolumn{6}{|c|}{ Primary diagnosis 1} \\
\hline Number & 0 & 2 & 0 & 31 & 107 \\
\hline Percent & & 1.4 & 0.0 & 22.1 & 76.4 \\
\hline \multicolumn{6}{|c|}{ Primary diagnosis 2} \\
\hline Number & 106 & 8 & 10 & 2 & 14 \\
\hline Percent & & 23.5 & 29.4 & 5.9 & 41.2 \\
\hline \multicolumn{6}{|c|}{ Primary diagnosis 3} \\
\hline Number & 136 & 0 & 2 & 0 & 2 \\
\hline Percent & & 0.0 & 50.0 & 0.0 & 50.0 \\
\hline \multicolumn{6}{|c|}{ Secondary diagnosis 1} \\
\hline Number & 96 & 3 & 14 & 2 & 25 \\
\hline Percent & & 6.8 & 31.8 & 4.5 & 56.8 \\
\hline \multicolumn{6}{|c|}{ Secondary diagnosis 2} \\
\hline Number & 127 & 0 & 2 & 0 & 11 \\
\hline Percent & & 0.0 & 15.4 & 0.0 & 84.6 \\
\hline \multicolumn{6}{|c|}{ Secondary diagnosis 3} \\
\hline Number & 132 & 0 & 3 & 0 & 5 \\
\hline Percent & & 0.0 & 37.5 & 0.0 & 62.5 \\
\hline \multicolumn{6}{|l|}{ Total } \\
\hline Number & 597 & 13 & 31 & 35 & 164 \\
\hline Percent & & 5.3 & 12.8 & 14.4 & 67.5 \\
\hline
\end{tabular}

Note. Information missing on both data sets has been excluded from calculation of percent 
Table 4

Agreement between MHMIS and client charts on ICD-9-CM psychiatric diagnosis at the three digit level

\begin{tabular}{|c|c|c|c|c|c|}
\hline \multirow[b]{2}{*}{ Diagnostic field } & \multirow[b]{2}{*}{$\begin{array}{c}\text { Both } \\
\text { missing }\end{array}$} & \multicolumn{3}{|c|}{ Disagree } & \multirow{2}{*}{$\begin{array}{c}\text { Agree } \\
\text { Chart \& } \\
\text { MHMIS } \\
\text { match }\end{array}$} \\
\hline & & $\begin{array}{c}\text { MHMIS } \\
\text { only }\end{array}$ & $\begin{array}{l}\text { Chart } \\
\text { only }\end{array}$ & $\begin{array}{l}\text { Chart \& } \\
\text { MHMIS } \\
\text { disagree }\end{array}$ & \\
\hline \multicolumn{6}{|c|}{ Primary diagnosis 1} \\
\hline Number & 0 & 2 & 0 & 22 & 116 \\
\hline Percent & & 1.4 & 0.0 & 15.7 & 82.9 \\
\hline \multicolumn{6}{|c|}{ Primary diagnosis 2} \\
\hline Number & 106 & 8 & 10 & 1 & 15 \\
\hline Percent & & 23.5 & 29.4 & 2.9 & 44.1 \\
\hline \multicolumn{6}{|c|}{ Primary diagnosis 3} \\
\hline Number & 136 & 0 & 2 & 0 & 2 \\
\hline Percent & & 0.0 & 50.0 & 0.0 & 50.0 \\
\hline \multicolumn{6}{|c|}{ Secondary diagnosis 1} \\
\hline Number & 96 & 3 & 14 & 1 & 26 \\
\hline Percent & & 6.8 & 31.8 & 2.3 & 59.1 \\
\hline \multicolumn{6}{|c|}{ Secondary diagnosis 2} \\
\hline Number & 127 & 0 & 2 & 0 & 11 \\
\hline Percent & & 0.0 & 15.4 & 0.0 & 84.6 \\
\hline \multicolumn{6}{|c|}{ Secondary diagnosis 3} \\
\hline Number & 132 & 0 & 3 & 0 & 5 \\
\hline Percent & & 0.0 & 37.5 & 0.0 & 62.5 \\
\hline \multicolumn{6}{|l|}{ Total } \\
\hline Number & 597 & 13 & 31 & 24 & 175 \\
\hline Percent & & 5.3 & 12.8 & 9.9 & 72.0 \\
\hline
\end{tabular}

Note. Information missing on both data sets has been excluded from calculation of percent 


\section{Table 5}

Source of disagreement between MHMIS and client charts on primary diagnosis

\begin{tabular}{|c|c|c|c|c|}
\hline Source of error & $\begin{array}{c}\text { Number }(\%) \\
\text { cases }\end{array}$ & $\begin{array}{c}\text { Incorrect } \\
\text { identification }\end{array}$ & Case missed & $\begin{array}{l}\text { Noncase } \\
\text { identified }\end{array}$ \\
\hline
\end{tabular}

Entry error

$\begin{array}{lrr}\mathrm{N} & 1 & 1 \\ \text { \% disagree } & (4.5) & (4.5) \\ \text { \% total charts } & (0.7) & (0.7)\end{array}$

Diagnostic conflict

$\begin{array}{lrr}\mathrm{N} & 3 & 3 \\ \% \text { disagree } & (13.6) & (13.6) \\ \% \text { total charts } & (2.1) & (2.1)\end{array}$

MHMIS not updated

$\begin{array}{lrrrr}\mathrm{N} & 18 & 7 & 7 & 4 \\ \text { \% disagree } & (81.8) & (31.8) & (31.8) & (18.2) \\ \text { \% total charts } & (12.9) & (5.0) & (5.0) & (2.9)\end{array}$

Total

$\begin{array}{lrrrr}\mathrm{N} & 22 & 10 & 7 & 4 \\ \% \text { disagree } & (100) & (45.5) & (31.8) & (18.2) \\ \text { \% total charts } & (15.7) & (7.1) & (5.0) & (2.9)\end{array}$


of the change which is not reported. A case which is opened with a nonspecific diagnostic code such as '7999' (not yet diagnosed) who later receives a diagnosis that is not entered represents a true case which is missed by MHMIS $(\mathrm{N}=7)$. Conversely, a case opened with a diagnosis based on referral information may have no pathology found in the assessment. If the new information is not submitted, a noncase appears as a case in MHMIS $(\mathrm{N}=4)$. A diagnosis can also be changed over the course of treatment. If the most recent information is not captured in MHMIS then the case is incorrectly identified $(n=7)$.

Comorbid diagnoses. Primary diagnosis must be captured in MHMIS and is usually recorded in the client chart. Comorbid diagnoses are optional and can be captured in one set of records and missed on the other set of records. For instance, a client seen for depression with concurrent substance abuse may have only depression captured in MHMIS. Similarly, MHMIS may contain codes for conditions not noted in the chart.

A total of 31 diagnoses (24 individuals) found in client charts were not recorded in MHMIS. Eleven of these result from failure to update client charts. These are the same individuals that were identified in discussion of primary diagnosis. Failure to update MHMIS affects all diagnoses for that individual. The client charts for the remaining 13 individuals contain information that is potentially valuable and is not captured in MHMIS. It was anticipated that this 
phenomenon would predominantly affect regional services charts since the regions typically submit only the required primary diagnosis. However, the missing cases were evenly distributed across all region/facilities.

MHMIS contains 11 diagnoses (10 individuals) that were not recorded on client charts. Three of these cases resulted from failure to update MHMIS as discussed previously. The remaining eight diagnoses were all V codes.

Agreement between chart and MHMIS is not substantially improved by using hierarchical categories. Table 6 shows agreement between chart and MHMIS on mental health categories. Agreement on primary diagnosis is increased to 85 percent from 82.9 percent at the three digit level. When hierarchically categorizing all diagnoses, agreement increases to 73.6 percent from 72.1 percent on all diagnoses.

Insert Table 6 about here

General linear modelling shows that there are no differences in agreement on primary diagnosis at the three digit level related to service type, clinician type or region $\backslash$ facility $(F=1.39$ with 16,123 df $p<0.16)$.

\section{Issues Regarding Diagnosis}

Clinicians may have very different views about the diagnosis for a 
Table 6

Agreement between MHMIS and client charts using hierarchical mental health categories

\begin{tabular}{lccccc}
\hline Client Charts & \multicolumn{5}{c}{ MHMIS } \\
\cline { 2 - 6 } & Psychotic & Nonpsychotic & Substance & Other & Not \\
& 47 & 3 & abuse & & classified \\
Psychotic & 1 & 26 & 0 & 1 & 3 \\
Nonpsychotic & 0 & 0 & 0 & 0 & 6 \\
Substance abuse & 0 & 1 & 0 & 0 & 0 \\
Other & 0 & 3 & 0 & 18 & 1 \\
Unclassified & 0 & & 0 & 2 & 22 \\
\hline
\end{tabular}


particular client and very different approaches to treatment. An individual presenting with depression and marital discord may receive a diagnosis of depression from a physician or psychiatrist while another clinician may identify marital discord as the issue. In still other circumstances, the depression may be emphasized in order ensure the client has access to services. While all the clinicians who were interviewed felt that the diagnoses they provide accurately reflect the client, it is also clear that diagnosis could be more precise. Whether from concern over labelling clients, ensuring access to service, or discomfort with making diagnoses, many diagnoses are more general than they need to be.

Agreement on diagnosis between research scientists from the same discipline is limited. Agreement on diagnosis by a range of clinicians from different professional backgrounds and differing levels of comfort with diagnosis is likely to be considerably lower. Investigation of the validity of a given diagnosis is beyond the scope of this investigation, but information on validity would certainly be of value in ascertainment of the research potential of any data base.

\section{Contact Information}

A contact (or encounter) refers to a significant therapeutic contact with or regarding a client, either in person or by phone. The Encounter form captures information on the date, type of contact (face to face or by phone, with client or 
collateral, scheduled or unscheduled as well as missed appointments) and information on services or therapies provided.

Encounter forms were originally designed to be client specific. A form was to be kept for each client, the forms were to be maintained in the client chart and completed forms were to be sent to clerical staff for entry. This practice was unwieldy since some clients are seen infrequently and the data may not be entered for a lengthy period. In addition, it was difficult to ensure that data for all clients was entered. In response to these difficulties, all regions/facilities in the study have developed their own forms. The revised Encounter forms contain information on all contacts made by a single clinician with all clients for a given time period. Upon completion, the forms are sent to clerical staff for entry but are not retained in client charts since they are not client specific.

\section{Detailed Methods for Contact Information}

All client contacts noted in the client chart in the period from May 1, 1994 to April 30, 1995 or during the episode of care if the case was open less than one year were recorded and compared to MHMIS information for the same period. Although MHMIS can not contain contacts outside the dates a case is open, contacts identified in the client chart either before the case was open or after the case was closed were recorded. In the event that a case was closed and reopened during the course of the year, only contacts related to the sampled episode were 
recorded. Contact information for inpatients is not submitted by some clinicians. Because of this, inpatient data is excluded from the analysis of contact information.

Unique contact dates were used rather than unique contacts since it is not always possible to determine what action or series of actions took place. This impacts on both client charts and on information submitted to MHMIS. For instance, several client contacts on a single day may result in a single chart notation or a single contact may result in more than one notation. Similarly, a number of different therapies may be provided and entered into MHMIS as separate contacts even though they were all part of the same contact. Therefore only unique dates of contact were examined.

Contacts which were identified in the client charts and could not be quantified were excluded. More specifically, five charts containing phrases such as 'work was done over the summer' or 'seen several times' were affected. Since the number of contacts cannot be determined, this information is lost. If charts contained information to the effect that a client was seen biweekly or 'twice since last note', the probable contact dates were recorded.

On a few client charts, reference is made to participation in another program such as supported employment or community based groups. Since these programs are community based initiatives, the dates of contact are not recorded 
in client charts nor is information submitted to MHMIS. Therefore, this information was not recorded. Programs delivered by the facility or region (such as adult or adolescent day treatment programs) and recorded in the client chart were recorded as client contacts.

General Linear Modelling was used to determine whether differences in agreement existed related to clinician type, region/facility or type of service. The proportion of agreement on specific encounter dates was used as the dependent variable. The number of contacts contained in both client charts and MHMIS as well as the amount of unique information in each data source were identified. Precise agreement between client charts and MHMIS was assessed, as well as the correlation between the quantity of information found in the two files. Correlation between contacts found in MHMIS and client charts was calculated using Pearson's Product Moment correlation coefficient.

\section{$\underline{\text { Results for Contact Information }}$}

Client charts for 100 outpatients and regional services clients contained a total of 984 contact dates while MHMIS showed 950 contact dates for these same clients. The number of contact dates found in client charts ranged between zero and 258 with 95 percent of charts showing fewer than 33 contact dates. The data is not normally distributed. As is frequently found with health services utilization data, most cases had only a few contacts in this period and 75 percent had fewer 
than 10 contacts. The individual with 258 contact dates is attending an adult day treatment program. The range and distribution of MHMIS contact dates is similar with a range between zero and 210 contacts.

Ten of the 100 client charts included in this analysis showed no contact within the specified period of time. Of these, six also showed no contact on MHMIS and the remaining four showed only a small number of contacts in MHMIS. In contrast, seven client charts which contained contact dates had no MHMIS contact dates.

GLM identified no differences in agreement based on a model including clinician type, service type and region/facility $(F=1.07$ with $14,85 \mathrm{df}, \mathrm{p}<$ 0.40). MHMIS contact dates match specific contact dates in client charts only 40.8 percent of the time (Table 7). Overall, overlap between chart and MHMIS identified contact dates is 48.6 percent $(\mathrm{N}=633)$ with 27 percent $(\mathrm{N}=351)$ contacts uniquely identified in client charts and 24.4 percent $(\mathrm{N}=317)$ contacts uniquely identified in MHMIS. These numbers include six cases that showed no contact on either data set.

Insert Table 7 about here

Correlation between client contacts identified in client charts and in 
Table 7

Agreement between MHMIS and client charts on client contacts by clinician type and region/facility of service

\begin{tabular}{|c|c|c|c|c|c|}
\hline \multicolumn{6}{|c|}{ Clinician Type } \\
\hline & Physician & \multicolumn{2}{|c|}{$\mathrm{RPN}^{\mathrm{a}}$} & Social & Other \\
\hline Number of cases & 22 & \multicolumn{2}{|c|}{45} & 20 & 13 \\
\hline Mean agreement & 0.58 & \multicolumn{2}{|c|}{0.37} & 0.38 & 0.29 \\
\hline & \multicolumn{5}{|c|}{ Region/Facility } \\
\hline & Facility 1 & Facility 2 & Region 1 & Region 2 & Region 3 \\
\hline Number of cases & 20 & 20 & 20 & 20 & 20 \\
\hline Mean agreement & 0.39 & 0.58 & 0.28 & 0.45 & 0.34 \\
\hline
\end{tabular}

Note. Based on the proportion agreement between data sources

a Registered Psychiatric Nurse 
MHMIS is shown in Table 8 . Overall correlation between the number of contacts identified in client charts and MHMIS was 0.963. This correlation is reduced to 0.814 if the outlier is removed.

Insert Table 8 about here

\section{Issues Regarding Contact Information}

During the interviews, clinicians identified that recording is often completed some time after the client is seen, perhaps even weeks later. The date of notations in the client chart usually refers to date of recording rather than the date the client was actually seen. While a few charts specifically recorded dates of client contact in the note, most do not record this information. As a result comparison of precise dates has some limitations. Visual inspection of the contact dates in client charts and in MHMIS showed that the distance between contacts varied widely, suggesting that use of an algorithm allowing agreement within a few days would be of little benefit.

Although no significant differences were found between region/facilities, clinician types or service type, certain factors identified in the interviews seemed to be related to either higher or lower levels of agreement. Clerical staff transcribe dictation and submit MHMIS contact information for physicians. At 
Table 8

Correlation between client contacts identified by MHMIS and client charts

\begin{tabular}{|c|c|c|c|c|c|c|}
\hline & \multicolumn{6}{|c|}{ Clinician Type } \\
\hline & Physician & $\mathrm{RPN}^{\mathrm{a}}$ & \multicolumn{2}{|c|}{ Social Worker } & Other & Total \\
\hline Number of cases & 22 & 45 & & 20 & 13 & 100 \\
\hline Including outlier & 0.994 & 0.652 & & 0.907 & 0.754 & 0.963 \\
\hline \multirow[t]{3}{*}{ Excluding outlier } & 0.874 & 0.652 & & 0.907 & 0.754 & 0.814 \\
\hline & \multicolumn{6}{|c|}{ Region/Facility } \\
\hline & Facility 1 & Facility 2 & Region 1 & Region 2 & Region 3 & Total \\
\hline Number of cases & 20 & 20 & 20 & 20 & 20 & 100 \\
\hline Including outlier & 0.993 & 0.899 & 0.734 & 0.906 & 0.704 & 0.963 \\
\hline Excluding outlier & 0.922 & 0.899 & 0.734 & 0.906 & 0.704 & 0.814 \\
\hline
\end{tabular}

a Registered Psychiatric Nurse 
interview the clerical staff indicated that some cross-checking is done to ensure that notes and MHMIS entries match. This practice appears to increase the agreement between data sources since agreement was highest for physicians. The outpatient service with a concentration of physician/psychiatrists also showed higher agreement.

Agreement between sources is decreased when block charting is used. For instance, one region that places assessments and periodic updates on the chart but does not encourage routine documentation of contacts had lower levels of agreement between data sources. In this instance, MHMIS provides a better source of information than client charts.

A discrepancy was noted in the practice of recording contacts for day treatment programs. One day treatment program enters each client attendance as a contact while another day treatment program operated by the same facility does not enter this data. MHMIS documentation does not specifically address whether this information should or should not be entered. It is not known how many programs may be affected.

In the clinician interviews and in the preliminary interviews it was identified that some clinicians submit little or no contact information. In addition, some regions have tolerated widespread noncompliance with recording of contact data. Based on collateral information, noncompliance with submission of contact 
information may even have increased in the interval following the study period. Since this study involved reviewing only a small number of charts for each clinician, it is not possible to determine whether clinicians in this sample were among those who do not submit information. This is an area that warrants further investigation with a more suitable sample.

\section{Open and Close Dates}

The open and close dates entered into MHMIS describe the temporal boundaries of service delivered to a client by a specific facility or region. The open date is the date when formal service is initiated and the close date is the date on which formal mental health services are terminated (MHMIS, 1991). The open and close dates then are potential proxies to describe an episode of care.

\section{Detailed Methods for Open and Close Dates}

The decision as to when formal services have been initiated or terminated is made by the clinician. Some clinicians open a case based on a referral or a significant telephone conversation, while others do not open a case until an intake assessment has been conducted and the client has been assigned to a clinician. Termination of a case may be documented in the client chart with a note stating that the case is closed. In other cases the date of closure is less clear since the client simply stops attending and the case is closed after a period of no contact. For this analysis, the open date attributed to a client chart is the date of the 
earliest documentation on the client chart. The date could therefore be for receipt of the referral, intake call or for the initial contact with the client. The last date of documentation was considered to be the close date.

The sampling method allowed for review of both open and closed cases. Cases were considered to be closed if they stated that the case was closed, if a referral was made to another organization that was taking over responsibility for the case, or if the case had been inactive for some period of time. The period of time required for a case to be considered closed depended on the individual case. For instance, if a client was generally seen yearly for assessment under an order of supervision, and was seen only once during the year the case would be considered open but a case would be considered closed if, for example monthly contact with the client suddenly stopped and there was no further documentation for six months.

For open date and close date, the fit between MHMIS and chart data was assessed by identifying the number of days difference between MHMIS and chart dates. Cases which were very poorly matched such as cases with more than one year between chart and MHMIS dates were inspected to see whether there were common reasons for a poor match.

Differences in agreement based on region/facility or type of service or clinician type were investigated using GLM. In order to examine whether 
differences existed between clinician types, region/facility or type of service, the proportion of agreement on open and close was used. The proportion for open cases would be based only on the match for open date. The proportion for closed cases would be based on the match for both open and close dates.

Contacts found in the client charts which were outside the MHMIS open and close dates were inspected to determine the extent to which MHMIS open and close dates accurately capture an episode of care. Since information on client contacts was not recorded for inpatients, investigation of this question was limited to outpatients and regional services clients.

\section{$\underline{\text { Results for Open and Close Dates }}$}

According to client charts, seventy four of the 140 cases which were reviewed were closed and 66 cases were open. Sixty-seven percent of reviewed cases had an exact match between MHMIS and client charts on the date a case is opened (see Table 9). An additional 15 percent are in agreement within one week. Ten cases disagreed by more than one year. Of the cases that disagreed by more than one year, one had no open date on the chart and one case was opened to the outpatient department on discharge from inpatient services but the client never subsequently used the services. The case was closed by clerical staff three years later. Four additional cases from a single facility were opened to MHMIS in June and July of 1990 but were long term cases at that point. Since 
this is the time period when MHMIS was being implemented, it is likely that the date used to open the cases was the date of entry rather than the date the client started receiving services.

Insert Table 9 about here

For closed cases, agreement on the date when formal services are terminated is very similar to agreement on open date. Two of the three cases which disagreed on close were clients that had not been seen in three years. In one case, the MHMIS Close form had been completed and was on the client chart but the information had not been entered into MHMIS. The third case had been closed by the clinician as of the correct date but the information had not yet been entered into MHMIS. Specifically, the client was seen up until April of 1994 but the close information, dated April 1994, was not submitted until May of 1995.

In cases where the dates disagree, the client chart is more likely to open first and close first (76\% and $69 \%$ respectively). For the most part this is a bias of the operational definition of opened utilized for the chart dates. Thus activity in the client charts prior to opening a case was usually limited to receipt of referral, phone calls to set up appointments or to obtain collateral information. However, one or two client contacts occurred prior to opening the case in 
Table 9

Proximity of MHMIS open dates and close dates to client chart information on open and close

\begin{tabular}{|c|c|c|c|c|c|c|}
\hline \multirow{3}{*}{ Agreement period } & \multirow{3}{*}{$\mathrm{N}$} & \multicolumn{2}{|c|}{ Open date } & \multirow{3}{*}{$\mathrm{N}$} & \multicolumn{2}{|c|}{ Close date } \\
\hline & & Percent & Cumulative & & Percent & Cumulative \\
\hline & & agreement & percent & & agreement & percent \\
\hline Exact agreement & 94 & 67.1 & 67.1 & 51 & 68.9 & 68.9 \\
\hline Within one week & 21 & 15.0 & 82.1 & 09 & 12.2 & 81.1 \\
\hline Within one month & 7 & 5.0 & 87.1 & 3 & 4.1 & 85.1 \\
\hline Within one year & 8 & 5.7 & 92.9 & 8 & 10.8 & 95.9 \\
\hline More than one year & 10 & 7.1 & 100.0 & 3 & 4.1 & 100.0 \\
\hline Total & 140 & 100.0 & 100.0 & 74 & 100.0 & 100.0 \\
\hline
\end{tabular}

Note. Close date excludes cases that were open at the end of the study period. 
MHMIS in five cases. Three cases had client activity in the chart after the case was closed. In one of these cases the activity was a termination summary but the other two charts showed several client contacts (three and six).

GLM identified significant differences in agreement on open date and close date for a model including service type, clinician type and region/facility $(\mathrm{F}=$ 3.51 with $16,123 \mathrm{df}, \mathrm{p}<0.0001$ ). Both service type and clinician type were identified as being significant $(F=15.65$ with $2,123 \mathrm{df} p<0.0001$ and $\mathrm{F}=$ 5.08 with $3,123 \mathrm{df} p<0.024$ respectively). A Duncans multiple range test ( $\mathrm{p}$ $=0.05)$ showed that inpatient services had significantly higher agreement than regional or outpatient services which did not differ from one another. This is predictable since open and close dates for inpatient services are easily identified (see Table 10). The Duncans multiple range test failed to identify differences between different types of clinician.

Insert Table 10 about here

\section{Issues Regarding Open and Close Dates}

Both open and close information can be retrospectively entered in MHMIS. If information is submitted a long time after the event has occurred, the accuracy of information taken off the system at a given point may be 
Table 10

\section{Agreement between MHMIS and client charts on open and close dates}

Type of service

Inpatient services Regional services Outpatient services

$\begin{array}{lccc}\text { Number of cases } & 40 & 60 & 40 \\ \text { Mean agreement } & 1.0 & 0.708 & 0.700\end{array}$

Clinician type

Physician/ Other RPN ${ }^{\mathrm{a}} \quad$ Social Worker

Psychiatrist

Number of cases

62

13

45

20

Mean agreement

0.815

0.808

0.789

0.700

Note. Based on proportion agreement on dates within one month. A line underneath the column indicates no significant difference between groups according to Duncans multiple range test $(\mathrm{p}=$ $0.05)$.

${ }^{a}$ Registered Psychiatric Nurse 
compromised. Delays of a month or two may not have much impact, but lengthy delays impact on information available for research. Such lengthy delays were found in only one of the 140 reviewed cases $(0.71 \%)$.

Receipt of regular case lists would possibly be of benefit in identifying cases which need to be closed. For instance, the appearance of a case on a clinicians caseload after the clinician believes it to be closed is likely to prompt investigation. If the clinician believes a case has been closed and does not receive a client list, there is no means to identify that action to close the case is required.

In reviewing the client charts it appeared that a large number of regional and outpatient clients were not seen for an extended period of time prior to closure. Six or more months without contact was not uncommon. Although it would be of value to explore the length of time between the date the client is last seen an the date of closure, exploration of this question is beyond the scope of this study. Closed cases in regional and outpatient services would have to be reviewed for at least one year prior to closure. This study reviewed cases for one calender year rather than for a specific period prior to closure.

Apart from agreement between MHMIS and client charts on open and close dates, there are other important influences on use of these dates to define an episode of care. The dates of open and close of a case in MHMIS refer to the time that the client was receiving services from a particular type of service. For 
instance, the open and close date for inpatients describe the bounds of the period that a client was an inpatient while the open and close date for an outpatient describe the bounds of outpatient service. Since a client can not be open to both inpatient and outpatient services at the same time, an outpatient client who is admitted to the facility is closed to outpatient services. When the client is discharged, a separate outpatient case is opened. For instance, a client that is seen by outpatient services over a period of years with occasional hospitalizations appears to have a series of episodes of outpatient service. In order to define the period of time a client is receiving mental health services, sequential episodes must be considered as part of a single episode of care. It is also important to note that the length of time between discharge and being seen by outpatient services varies between a few days and a few months, depending on the client. This may have less impact on regional services since a case can be open to a facility and a region at the same time.

\section{Overall Reliability of MHMIS and Chart Data}

\section{Detailed Methods for Overall Reliability}

Using the summary variable which was developed for the generalizability analysis, GLM was used to determine whether there were overall differences in agreement between data sources based on service type, clinician type or region/facility. The summary variable is a composite of agreement on 
demographic data, diagnosis, contact data and open and close date.

\section{Results for Overall Reliability}

Significant differences were found $(F=3.42$ with $16,123 \mathrm{df}, \mathrm{p}<$ 0.0001 ) with the model explaining 30.8 percent of the variation between data sources. Significant differences were found based on service type $(F=8.90$ with $2,123 \mathrm{df}, \mathrm{p}<0.002$ ) but no other variables were significant (Table 11). A Duncans multiple range test $(\mathrm{p}=0.05)$ showed that inpatient data had higher levels of agreement than outpatient and regional services, which did not differ from one another (Table 12). Since differences based on region/facility were not found for any of the variables separately or for the data as a whole, and since maximally different regions were selected for review, it is unlikely that collecting data from the additional facility and regions would have provided different information.

Insert Table 11 and 12 about here

\section{Issues Regarding Overall Reliability}

Since region/facility is only partially nested within service type, a separate analysis was conducted to determine whether considering region/facility separately 
Table 11

Impact of service type, region/facility and clinician type on overall differences in agreement between MHMIS and client charts

\begin{tabular}{lrrrrr}
\hline Source of error & df & SS & MS & F & Pr > F \\
\hline Service type (S) & 2 & 0.4540 & 0.2270 & 8.90 & 0.0002 \\
Region/facility * (R) nested within S & 4 & 0.1493 & 0.0373 & 1.46 & 0.2173 \\
Clinician type (C) & 3 & 0.0787 & 0.0262 & 1.03 & 0.0382 \\
$\mathrm{~S} * \mathrm{C}$ & 3 & 0.0128 & 0.0043 & 0.17 & 0.9186 \\
$\mathrm{R} * \mathrm{C}$ nested within S & 4 & 0.0520 & 0.0130 & 0.51 & 0.7288 \\
Error & 123 & 3.1358 & 0.02550 & & \\
\hline Total & 139 & 4.5320 & & & \\
\hline
\end{tabular}


Table 12

Overall agreement between MHMIS and client charts based on type of service

Service type

Inpatient services Outpatient services Regional services

Number of cases

Mean agreement
40

0.883
40

0.721
0.672

60

Note. A line underneath the column indicates no significant difference between groups according to Duncans multiple range test $(\mathrm{p}=0.05)$. 
would alter the results. The overall significance of the model and amount of variation explained remain the same. Significance for service type remains high ( $F=20.98$ with $1,123 \mathrm{df}, \mathrm{p}<0.0001$ level) and no other changes occur.

\section{ISSUES AFFECTING THE INFORMATION AS A WHOLE}

While minimal differences in accuracy of MHMIS information were found between regions, the mental health services delivered in each region differ dramatically. Based on charts reviews, it appears that one region provides primarily crisis intervention. The typical client is seen the day they contact the service, the client is seen one or two more times and then does not return. Diagnosis is often 7999 (not yet diagnosed). The case is changed to maintenance after a time and is then closed. The second region provides planned services to individuals with anxiety and depression. Clients are typically seen within one week of intake, go through a series of sessions and are closed. Clients in the third region are usually suffering from psychotic conditions such as schizophrenia and bipolar disorder. It can take a full month between referral and the date when the client is first seen and clients are usually on the case load for a very long period of time. The finding that persons with psychotic disorders are concentrated in an urban center is consistent with earlier work by Tataryn, Mustard and Derksen (1994).

While each region provides a range of services, the services that are 
provided are related to the needs of the population and the other services that are available. If there are few individuals with a psychotic disorder in the region and if there are no alternate crisis intervention services, regional services will meet the needs of the community for crisis services. In a region with a high number of individuals suffering from psychotic disorders and where alternate crisis services are available, regional services again meet the needs of the population. However, the actual services delivered differ dramatically. As a result, comparison of caseloads, length of time in service, frequency of contact with clients and so forth across regions is not informative and could lead to erroneous conclusions.

Similarly, clinicians within a region/facility are often assigned cases based on their area of expertise. Therefore certain clinicians caseloads may contain a concentration of clients with a specific diagnosis. While this method of assigning cases effectively meets client needs, it makes comparison of the type and number of clients served meaningless.

Most MHMIS information is submitted within days or weeks of the event with some information being submitted on a monthly basis. However, a small amount of information is not submitted to MHMIS for a few months. For instance, delays were identified in entering diagnosis at one facility, and in opening cases to regional clinicians working in suboffices in one region. Cases 
that are closed as of an earlier date may also be affected. The delay in submitting these pieces of information does not necessarily influence the accuracy of the information. However, MHMIS data will be incomplete for information is submitted more than three months after the end of the fiscal year.

MHMIS contains a number of optional data fields. This information is collected routinely by some clinicians and not at all by others. Specific fields relevant to the region/facility may be captured in one area and not in another. The burden of paperwork can be substantial and it is important to know that only information that is needed is being collected. If information in the optional fields is needed, then this information should be required. If the information is not needed then it should not be collected. Partial information is of very little use.

MHMIS is not a static system. Both MHMIS itself and the environment in which it operates are dynamic. Changes in the available codes and in the how data is handled have taken place over time. Further refinements are sought by individuals who would like to be able to use the system more efficiently. The current movement toward regional governance and information based planning have placed demands on the system which is may not be able to meet. For instance, retrieval and manipulation of information at the regional level to allow planning is not currently possible. Future developments such as regional responsibility for information systems may impact on the quality and quantity of 
information in MHMIS. However, many of the issues which were identified will impact on the accuracy of any mental health information system.

\section{FACTORS INFLUENCING ACCURACY}

The intent of this research is to describe the reliability of MHMIS data rather than to suggest alternate methods of data collection. However, certain factors in data processing appear to influence data accuracy. Since accurate information is required for management as well as for research, it may be worthwhile to give consideration to these factors.

Clinicians generally considered record keeping to be an important part of working with clients and most indicated that they attempt to provide information that is as accurate as possible. Policies and procedures that facilitate record keeping are likely to enhance data accuracy. Integration of MHMIS data collection into other paperwork and streamlining the process of data collection are some of the ways in which data collection can be facilitated. Feedback mechanisms, including use of MHMIS information and regular review of clients are of benefit in keeping the information current.

Including MHMIS information on the assessment form makes it easier for individuals abstracting information to locate the information and make it more likely the information is recorded and entered. For instance, if religion or occupation is collected by the clinician and entered in an easily accessible location 
such as the assessment form, the information is more likely to be entered into MHMIS. Clinicians themselves are more likely to collect information they are prompted to collect. Some clinicians try to streamline the process of data collection by filling out the MHMIS form as part of the intake process, and using the form to look for specific pieces of information. However, MHMIS Open/Close form does not capture the full range of information usually collected at intake necessitating use of more than one form. Ideally, each region or facility could use a single form containing all the information required by both MHMIS and the region/facility.

Written rather than coded information on the forms or easy access to the codes may be of benefit to clinicians. Rather than going to the very large and centrally located MHMIS manual to select codes, one clinician prepared a few sheets with the codes for demographic variables, referral source and so forth. The sheets are posted by his desk so that he can refer to them and ensure accuracy rather than trying to remember the codes or using a favourite few.

Feedback mechanisms such as case lists, lists of inactive cases and routine review of cases increases accuracy because each of them causes the clinician to review the existing information and modify the information if it is incorrect. Since failure to update information such as diagnosis was one of the main reasons for disagreement on diagnosis, regular review and update of information could 
contribute significantly to accuracy of MHMIS data. Similarly, review of cases open to the clinician encourages prompt reporting of changes in clinician and closure of inactive cases. For clinicians who felt that the information was not used and was therefore unimportant, feedback of information would provide assurance that the data being collected is of value.

Completion of paperwork takes time. Clinicians tended to invest more in submitting complete and accurate information in regions/facilities where the supervisors considered documentation to be an important part of the work.

Information that is seen as useful is more likely to be accurate. Increased access to MHMIS and awareness of potential uses are necessary for increased use among clinicians. Those who used the information found it quite helpful but many did not know what information was available or how to access it. Managers who have tried to use MHMIS have generally been very frustrated by their inability to obtain information that meets their needs. Since supervisory support for record keeping was an important factor in supporting data collection, a system that is responsive to regional or facility information needs is more likely to contain accurate information.

A number of nonphysician clinicians expressed discomfort with providing their diagnostic impression. Due to this discomfort, the diagnostic impression may be more general than it needs to be. Although each CMHW is offered a 
course on diagnosis, more information may be of assistance in increasing the precision of diagnosis. Clinicians who had more preparation were more comfortable with diagnosis. However, some clinicians may continue to assign general diagnoses based on concerns about labelling or concerns about individuals being denied treatment based on diagnosis.

\section{DISCUSSION}

The individuals who were interviewed believe the demographic information is as accurate as it can be. Required information is complete but optional information is not always present. Information on gender, date of birth and marital status in client charts is highly similar to the same information in MHMIS with agreement ranging from 83.6 to 100 percent. Agreement on postal code, which is important as it provides information on location of residence and can be used to identify socio-economic risk, is considerably lower $(59.3 \%)$.

Agreement on the optional variables of education, employment, occupation, treaty status and religion is lower than for required variables. Agreement on optional informaticn ranges from 45.5 to 73.5 percent if cases which are missing the information in both data sources are excluded.

The diagnosis assigned to clients appears to be quite subjective but in the view of the clinicians, the diagnoses supplied to MHMIS do reflect the client. This view is supported by chart reviews. In reviewing the client charts it was 
noted that the chart diagnosis was consistent with how the clinician described the client and how the client was treated in all but one case. For instance, a client that was diagnosed as suffering from depression would have information in the chart describing symptoms of depression and treatment for depression.

Agreement on primary diagnosis between MHMIS and chart diagnosis at the three digit level is 82.9 percent. The usual cause of disagreement results from failure to update MHMIS when diagnosis is changed. Both MHMIS and client charts contain unique information. Information on client charts which is not captured by MHMIS represents a reduction in available information on comorbid mental disorders. Agreement on all diagnosis is lower than for primary diagnosis alone due to unique information on comorbid conditions on each data set.

Using mental health categories to describe cases produces a small improvement in agreement, but at the cost of more detailed information. Using all diagnoses for classification produced substantially lower levels of agreement than primary diagnosis alone at the three digit level (73.6 percent and 82.9 percent respectively).

Clinicians report that MHMIS does not contain the information needed to examine clinician activity but information on client contact is captured accurately. Clinicians consistently report that virtually all direct client contact is captured 
although some phone calls and consultations may be missed. However, there is a suggestion from the interviews that there are individuals, programs and perhaps entire regions that do not submit contact information.

Specific contact dates are in agreement with chart dates less than 50 percent of the time. However, the correlation between the amount of data in the charts and in MHMIS is as high as 0.96. Despite this correlation, there are suggestions that data from some clinicians, programs and perhaps even regions may be missing. Contact information should be used with caution.

Clinicians felt that the open date accurately reflected the date when service was initiated but felt the close date did not reflect the termination of services. Status changes are rarely completed by clinical staff but may be submitted by clinical records and clerical staff in facilities. MHMIS information is used for a variety of purposes but it information is not as useful as people would like it to be.

GLM showed that there are no differences in agreement between MHMIS and chart information related to service type, provider type or region/facility for demographic information, primary diagnosis, or contact information. Differences in agreement were found for open and close dates and for overall agreement based on service type. Agreement is higher for inpatient services than outpatient or regional services although overall agreement is good in all cases. 


\section{CONCLUSION}

MHMIS data appears to be relatively accurate and complete. With the cautions noted above, much of MHMIS data can be used for research. Linkage of MHMIS data with hospital and physician claims in the Manitoba Health Research Data Base creates comprehensive information on the use of mental health services by a population of over one million people. The capacity of this combined system to track individuals over time and to maintain information on clients even when they are not receiving mental health services make this information a powerful instrument for psychiatric epidemiology as well as for planning, monitoring and evaluating mental health services. Given the magnitude of costs associated with treatment of mental disorders and the impact of mental disorder on the lives of affected individuals, this unobtrusive, cost-effective information should increasingly be used to improve our understanding or mental disorder and the impact of treatment and to guide decision making in mental health services. 


\section{REFERENCES}

Beecham, J., Knapp, M. \& Fenyo, A. (1991). Costs, needs, and outcomes. Schizophrenia Bulletin, 17 (3), 427-439.

Bell, J. F. (1985). Generalizability theory: the software problem. Journal of Educational Statistics. 10 (1) p 19-29.

Bergner, M. (1985). Measurement of health status. Medical Care, 23 (5), 696701.

Bigelow, D.A. (1989). State data systems and research opportunities. In J.D. Bloom (Ed.). State-university collaboration: the Oregon experience. New directions for health services No. 44 (pp 73-82). San Francisco, CA: Jossey-Bass.

Bigelow, D. A. \& Young, D. J. (1991). Effectiveness of a case management program. Community Mental Health Journal, 27 (2), 115-123.

Binner, P. R. (1993). Information systems and mental health services: Issues for the 90's. In Technology in people services (pp. 47-57). Haworth Press.

Bland, R.C. (1988). Prevalence of mental illness. Annals Royal College of Physicians and Surgeons of Canada, 21 (2), 89-93.

Brown, P. (1987). Diagnostic conflict and contradiction in psychiatry. Journal of Health and Social Behaviour, 28, 37-50.

Campbell, D.T., \& Fiske, D.W. (1959). Convergent and discriminant validation 
by the multi-trait-multimethod matrix. Psychological Bulletin, $\underline{56}$ (2), 81105.

Carmines, E.G., \& Zeller, R.A. (1979). Reliability and validity assessment. Beverly Hills, CA: Sage Publications.

Chapman, P. L. \& Ostye, T. (1987). The development of a mental health information system. New Zealand Medical Journal, 100, 287-289.

Cohen, J. (1968). Weighted Kappa: nominal scale agreement with provision for scales disagreement or partial credit. Psychological Bulletin, $\underline{70}$ (4), 213220.

D'Arcy, C. (1976). Patterns in the delivery of psychiatric care in Saskatchewan 1971-1972: An overview of service sectors and patient volumes. Canadian Psychiatric Association Journal, 21 (2), 91-100.

D'Arcy, C. (1977a). Patterns in the delivery of psychiatric care in Saskatchewan 1971-1972 (II): Types of contacts and some patient career characteristics. Canadian Psychiatric Association Journal, 22 (1), 31-36.

D'Arcy, C. (1977b). Patterns in the delivery of psychiatric care in Saskatchewan 1971-1972 (III): Patient socio-demographic and medical characteristics. Canadian Psychiatric Association Journal, 22 (5) 215-224

D’Arcy, C. \& Bold, G. (1983). Alcohol and drug dependency in Saskatchewan, 1969-1974. Journal of Studies on Alcohol, 44 (4), 630-646. 
Dickens, B. M. (1983). Political and legal considerations. In E. M. Bennett \& B. Trute (Eds.). Mental health information systems: Problems and prospects. (pp. 99-120). Toronto, ON: Edwin Mellen Press.

Fleiss, J.L. (1981). The measurement of interrater agreement. In Statistical methods for rates and proportions 2 nd ed. (pp.212-236). Toronto, ON: John Wiley \& Sons.

Fryers, T. (1987). The future of psychiatric case registers. In B. Cooper (Ed.) Psychiatric epidemiology (pp. 335-351). Croom Helm.

Hafner, H. \& Pfeifer-Kurda, M. (1986). The impact of data protection laws on the Mannheim case register. In G.H.M.M. ten Horn, R. Geil \& J. H. Henderson (Eds.). Psychiatric case registers in public health (p. 366-371). Amsterdam, The Netherlands: Elsevier Science Publishers.

Helgason, T. (1986a). The european experience. In G.H.M.M. ten Horn, R. Geil \& J. H. Henderson (Eds.). Psychiatric case registers in public health (p. 361-365). Amsterdam, The Netherlands: Elsevier Science Publishers. Helgason, T. (1986b). The Icelandic experience. In G.H.M.M. ten Horn, R. Geil \& J. H. Henderson (Eds.). Psychiatric case registers in public health (p. 372-375). Amsterdam, The Netherlands: Elsevier Science Publishers. Kirk, S.A., \& Kutchins, H. (1988). Deliberate misdiagnosis in mental health practice. Social Science Review, June, 225-237. 
Kirk, S.A., \& Kutchins, H. (1992). The selling of DSM: the rhetoric of science in psychiatry. New York, NY: Aldine de Gruyter.

Klerman, G.L., Olfson, M., Leon, A.C., \& Weissman, M.M. (1992). Measuring the need for mental health care. Health Affairs, Fall, 23-33.

Kutchins, H., \& Kirk, S.A. (1988). The business of diagnosis: DSM-III and clinical social work. Social Work, May-June, 215-220.

Last, J.M. (1988). A dictionary of epidemiology. Toronto, ON: Oxford University Press.

Lipton, A.A., \& Simon, F.S. (1985). Psychiatric diagnosis in a State hospital: Manhattan State revisited. Hospital and Community Psychiatry, $\underline{36}$ (4), 368-373.

Manitoba Health. (1992). Building the future of mental health services in Manitoba: a continuing partnership for reform. Winnipeg, MB: Author. Manitoba Health, Mental Health Division \& Manitoba Health Services Commission. (1991). Mental health management information system policy and procedure manual. Winnipeg, MB: Author.

Manitoba Health. (no date). Annual report 1993/94. Winnipeg, MB: Author.

Miller, G. H., \& Willer, B. (no date). Information systems for evaluation and feedback in mental health organizations. In Evaluation research methods (pp. 199-215). 
Mirowski, J. \& Ross, C. E. (1989). Psychiatric diagnosis as reified measurement. Journal of Health and Social Behaviour, $\underline{30}, 11-25$.

Mortensen, P. B. (1995). The untapped potential of case registers and recordlinkage studies in psychiatric epidemiology. Epidemiologic Reviews, $\underline{17}$ (1), 205-209.

Mossey, J. M. \& Roos, L. L. (1987). Using insurance claims to measure health status: The illness scale. Journal of Chronic Diseases, $\underline{40}$ (Suppl. 1), 415505.

Munk-Jergensen, P., Kastrup, M. \& Mortensen, P. B. (1993). The Danish psychiatric case register as a tool in epidemiology. Acta Psychiatrica Scandinavica, $\underline{370}, 27-32$.

Nutter, R.W. (1983). Information systems and social work practitioners. The Social Worker, $\underline{51}(1), 3-7$.

Patrick, D. P., Bush, J. W. \& Chen, M. M. (1973). Toward an operational definition of health. Journal of Behaviour and Social Behaviour, 14, 6-23.

Province of Manitoba (1994). Estimates of expenditure for the province of Manitoba. Winnipeg, MB: Author.

Romano, P.S., \& Luft, H.S. (1992). Getting the most out of messy data:problems and approaches for dealing with large administrative data sets. In M.L. Grady \& H.A. Schwartz (Eds.). Medical effectiveness research data 
methods (AHCPR Pub. No. 92-0056) (pp. 57-75). Rockville, MD: U.S.

Department of Health and Social Services.

Roos, L. L., Cageorge, S. M., Austin, E., \& Lohr, K. (1985). Using computers to identify complications after surgery. American Journal of Public Health. $\underline{75}$ (11), 1288-1295.

Roos, N.P., Montgomery, P., \& Roos, L.L. (1987). Health care utilization in the years prior to death. The Milbank Quarterly, $\underline{65}$, (2), 231-254.

Roos, L. L., Mustard, C. A., Nicol, J. P., McLerran, M. S., Malenka, D. J., Young, T. K. \& Cohen, M. M. (1993). Registries and administrative data: organization and accuracy. Medical Care. 31 (3) p 201-212.

Roos, L.L., Nicol, J.P., Johnson, C.F., \& Roos, N.P. (1979). Using administrative data banks for research and evaluation: a case study. Evaluation Quarterly, $\underline{3}(2), 236-255$.

Roos, L.L., Nicol, J.P., \& Roos, N.P. (1983) Using large-scale data banks: productivity and quality control. In E.M. Bennett \& B. Trute (Eds.). Mental health information systems: problems and prospects, (pp. 81-97). Toronto, On: Edwin Mellen Press.

Roos, L.L., \& Roos, N.P. (1989). Large databases and research on surgery. In I.M. Rutkow (Ed.) Socioeconomics of surgery (pp. 259-275). St. Louis: C.V. Mosby. 
Roos, L.L., Roos, N.P., Cageorge, S.M., \& Nicol, P. (1982). How good are the data? Reliability of one health care data bank. Medical Care, $\underline{20}$ (3), 266276.

Schwartz, A.H., Perlman, B.B., Martin, P., Schmidt, B.S., \& Thornton, J.C. (1980). Psychiatric diagnosis as reported to Medicaid and as recorded in patient charts. American Journal of Public Health, 70 (4), 406-408.

Sharfstein, S.S., Towery, O., \& Milowe, I. (1980). Accuracy of diagnostic information submitted to an insurance company. American Journal of Psychiatry, 137 (1), 70-73.

Shavelson, R.J., Webb, N.M., \& Rowley, G.L. (1989). Generalizability theory. American Psychologist, 44 (6), 922-932.

Snyder, S. \& Strain, J.J. (1990). Change in diagnosis at termination of psychiatric consultation. Hospital and Community Psychiatry, 41 (3), 286289.

Spitzer, R.L., Cohen, J., Fleiss, J.L., \& Endicott, J. (1967). Quantification of agreement in psychiatric diagnoses. Archives of General Psychiatry, 17, 83-87.

Studney, D.R., \& Hakistan, R. (1981). A comparison of medical record with billing diagnostic information associated with ambulatory medical care. American Journal of Public Health, 71 (2), 145-149. 
Tataryn, D.J., Mustard, C., \& Derksen, S. (1994). The utilization of medical services for mental health disorders Manitoba: 1991 - 1992. Winnipeg, MB: Manitoba Centre for Health Policy and Evaluation, University of Manitoba.

Tataryn, D.J., Roos, N., \& Black, C. (1994). Utilization of physician resources volume 1: key findings. Winnipeg, MB: Manitoba Centre for Health Policy and Evaluation, University of Manitoba.

Tefft, B. M. (1983). An administrative perspective. In E. M. Bennett \& B. Trute (Eds.). Mental health information systems: Problems and prospects. (pp. 35-58). Toronto, ON: Edwin Mellen Press.

ten Horn, G. H. H. M., Geil, R., \& Gulbinat, W. H.(Eds) (1986). Psychiatric case registers in public health: A worldwide inventory 1960-1985. Amsterdam, The Netherlands: Elsevier Science Publishers.

ten Horn, G.H.M.M. (1986). Definitions and classifications. In G.H.M.M. ten Horn, R. Giel, W.H. Gulbinat \& J.H. Henderson (Eds.). Psychiatric Case

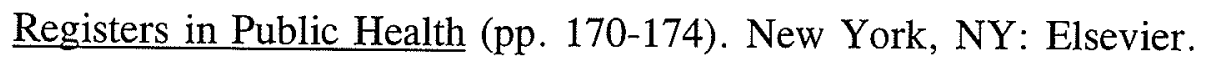

Trute, B. (1983). Human factors inherent in system survival. In E.M. Bennett \& B. Trute (Eds.). Mental health information systems: problems and prospects (pp. 71-80). Toronto, ON: Edwin Mellen Press.

Trute, B., \& Tonn, R. (1982). Privacy and computerized health information in 
community health centres and district health agencies. Medical Care, $\underline{20}$ (2), 202-215.

U.S. Department of Health and Human Services, (1986). Mental health program performance measurement. Rockville, MD: U.S. Government Printing Office.

Wing, J. K. (1983). Scientific issues. In E. M. Bennett \& B. Trute (Eds.). Mental health information systems: Problems and prospects. (pp. 15-33). Toronto, ON: Edwin Mellen Press.

Wing, J. K. (1986a). Data protection and problems of data confidentiality: Introduction. In G.H.M.M. ten Horn, R. Geil \& J. H. Henderson (Eds.). Psychiatric case registers in public health (p. 360). Amsterdam, The Netherlands: Elsevier Science Publishers.

Wing, J. K. (1986b). Safeguarding the patient and safeguarding research: The U.K. data protection act. In G.H.M.M. ten Horn, R. Geil \& J. H. Henderson (Eds.). Psychiatric case registers in public health (p 376-380). Amsterdam, The Netherlands: Elsevier Science Publishers.

Woogh, C.M. (1987). The case for psychiatric record linkage. Canadian Journal of Psychiatry, $32,470-475$.

Woogh, C.M. (1988). An experience in psychiatric record linkage. Canadian Journal of Psychiatry, $\underline{33}, 134-139$. 


\section{GLOSSARY}

Admission conference: A conference held within ten working days of admission to a mental health institution. Each discipline (nursing, occupational therapy, psychiatry, psychology and social work) prepares a report on the client, a diagnosis and treatment plan is formulated and an initial discharge plan is formed. Clinical records staff: Individuals employed specifically to maintain clinical records such as client charts and electronic information related to clients. These individuals may have specialized training. For instance, a Health Record Technician codes diagnosis on client charts.

Client chart: The paper record maintained by each region and each mental health facility containing information pertaining to a specific client.

Clinician: A mental health professional providing direct services to clients. Clinicians can include physician/psychiatrists, psychologists, RPNs, social workers or other providers.

Discharge conference: A conference held prior to discharge of a patient from a mental health institution. Each discipline (nursing, occupational therapy, psychiatry, psychology and social work) prepares a report on the client, a diagnosis and plan for aftercare is formulated.

Medical staff: Physicians or psychiatrists.

MHMIS data (or MHMIS information): Electronic records maintained by 
Manitoba Health containing information on services delivered to clients through Provincial mental health programs.

Inpatient: A person receiving services from a mental health facility on a residential basis.

Inpatient services: Residential treatment services provided in a mental health facility. In Manitoba inpatient services are provided through Brandon, Eden and Selkirk Mental Health Centres.

Outpatient: A person who receives services from a mental health facility on a nonresidential basis. This includes former inpatients who are seen for follow up and clients who have never been treated as inpatients.

Outpatient services: Clinical services provided by a mental health facility to clients who do not require residential treatment services. In Manitoba outpatient services are provided through Brandon, Eden and Selkirk Mental Health Centres. Regional services: Services delivered through multidisciplinary teams in each of Manitobas' eight health and social service regions. The community based services include assessment, treatment, rehabilitation and support. 


\section{Appendix A: MHMIS Open/Close Form}

MENTAL HEALTH

Maniloua
Heath

Client Open / Close Form

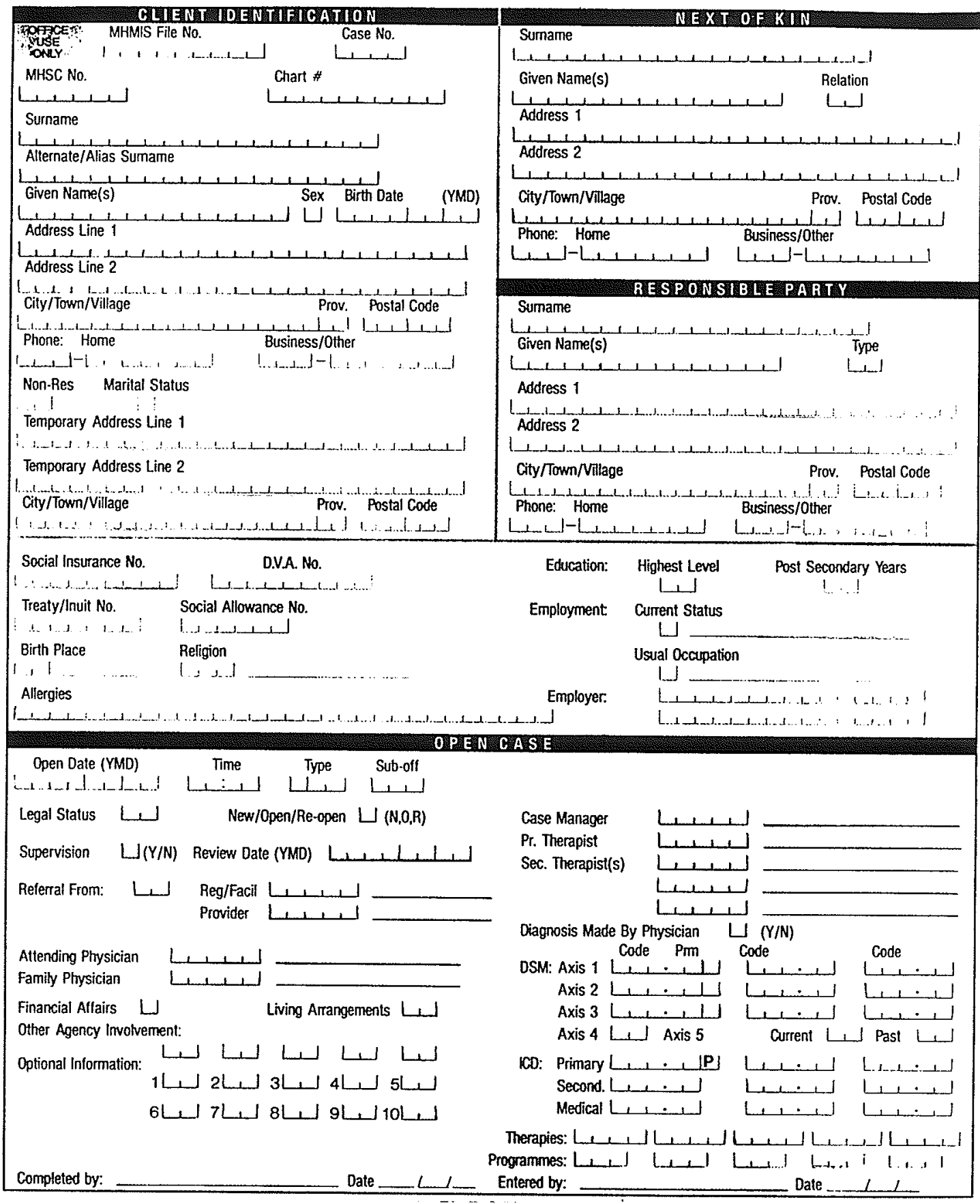




\section{Appendix B: Semi-structured Interview Schedule ${ }^{1}$}

\section{Section 1: Flow of information.}

* What MHMIS forms do you enter information on?

* What is the process for collecting and submitting information?

- Are standard assessment forms, periodic reviews or termination summaries part of your data collection?

* Is information documented on MHMIS forms that is not captured elsewhere on the client chart?

- How do you decide when to open a case and when to close a case?

- Are all clients that are seen opened as cases?

\section{Section 2: Beliefs about data accuracy.}

* Overall, how accurate do you consider MHMIS data to be?

* Are some data fields (demographic information, contact information, diagnosis or therapies information.

- Do you use diagnosis for purposes other than mandatory contribution to MHMIS? For example, is diagnosis useful in communicating about your client with colleagues or for discussing caseloads?

\section{Section 3: Factors influencing accuracy.}

* What factors do you feel influence the accuracy of the data collected?

- Do policies such as what clients can be served and when to open or close a case influence the information submitted?

- Do concerns result in alteration of data submitted.

* To what extent is the data influenced by each of these factors?

1 Questions are designed for use with clinicians. Due to differences in their role, clinical records and clerical staff were only asked those questions indicated with an asterisk. 


\section{Appendix C: Letter of Invitation}

Brandon, Manitoba

$\mathrm{R}^{\prime}$

August 31, 1995

\section{Dear Sir/Madam,}

I am a graduate student in the Department of Community Health Sciences at the University of Manitoba. I am writing to request your participation in a study expected to determine the reliability of the Manitoba Mental Health Management Information System (MHMIS) for research.

The project involves comparison of client charts and MHMIS data to determine agreement between the two sources of information as well as interviews with providers who contribute information to MHMIS. The interviews are expected to provide information on what factors influence completeness and accuracy of the data as well as the extent to which the data may be influenced.

Your participation in the study will enable me to determine which elements of MHMIS can be used for research, which elements can be used with caution and which elements should not be used. The information may also suggest ways in which data collection can be improved.

The project is being conducted in collaboration with, but independent of Manitoba Health. The project has support from the Mental Health Division and has been approved by the Medical Officers in Charge of Brandon, Eden and Selkirk Mental Health Centres as well as the Regional Directors in each region. At the end of the study, the results will be made available to Manitoba Health and to each region and facility.

Your name was drawn at random to take part in the interviews. I will be telephoning you in the next few days to give you further information and arrange a meeting.

I look forward to your participation in the study and your help in describing the quality of data in MHMIS. If you have any questions or concerns, please do not 
hesitate to contact me. I can be reached at

home
voice mail $\quad(\quad, \quad()$, or
email

Sincerely,

J. Renee Robinson, R.P.N., B.Sc. Mental Health. 


\section{Appendix D: Consent Form}

Study Title: Reliability of the Manitoba Mental Health Management Information System for Research.

Investigator: J. Renee Robinson, R.P.N., B.Sc. Mental Health.

The purpose of this study is to determine the reliability of the Manitoba Mental Health Management Information System (MHMIS) for research. Through these interviews Ms. Robinson hopes to determine what factors influence the completeness and accuracy of information in MHMIS. She also hopes to determine the extent to which the data is influenced by each factor.

Ms. Robinson is completing her Masters degree in the Department of Community Health Sciences at the University of Manitoba. This study is being conducted in cooperation with, but independent of, Manitoba Health.

Individuals who contribute information to MHMIS were randomly selected for interviews. In order to ensure that individuals being interviewed feel free to express their true feelings, numbers rather than provider name will be used on interview tapes. Results will be reported in aggregate only so that individual providers cannot be identified. Tapes of interviews and consent forms will be kept under double lock.

I understand that the study will involve an interview. I will also be asked to provide a diagnosis for one of my clients and to allow Ms. Robinson information from my appointment book. The interview will take about one hour and will be tape recorded. I understand I will not be paid for participation.

I have read the above description and voluntarily consent to participate in the study. I understand that my true identity will not be revealed to anyone other than the interviewer and that my comments will in no way affect my relationship with my employer. I am fully aware that I am free to decline participation at anytime.

If I have any questions about the study or about being a subject, I know I can call Ms. Robinson. I may reach her at ( )

Participants Signature

Date of Interview

Interviewers Signature 